\title{
Sharp Weighted convolution inequalities and some applications
}

\author{
WEICHAO GUO \\ School of Mathematics and Information Sciences \\ Guangzhou University \\ Guangzhou, 510006, P.R.China \\ E-mail: weichaoguomath@gmail.com \\ DASHAN FAN \\ Department of Mathematics \\ University of Wisconsin-Milwaukee \\ Milwaukee, WI 53201, USA \\ E-mail: fan@uwm.edu \\ HUOXIONG WU \\ School of Mathematical Sciences \\ Xiamen University \\ Xiamen, 361005, P.R.China \\ E-mail: huoxwu@xmu.edu.cn \\ GUOPING ZHAO \\ School of Applied Mathematics \\ Xiamen University of Technology \\ Xiamen, 361024, P.R.China \\ E-mail: guopingzhaomath@gmail.com
}

$\|$

\begin{abstract}
In this paper, the index groups for which the weighted Young's inequalities hold in both continuous case and discrete case are characterized. As applications, the index groups for the product inequalities

2010 Mathematics Subject Classification: Primary 42B15; Secondary 42B35.

Key words and phrases: weighted convolution inequalities, fractional integrals, discrete analogue, characterization, modulation spaces.
\end{abstract}


on modulation spaces are characterized, we also obtain the weakest conditions for the boundedness of bilinear Fourier multipliers on modulation spaces in some sense. For the fractional integral operator, the sharp conditions for the boundedness of power weighted $L^{p}-L^{q}$ estimates in both continuous case and discrete case are obtained. By a unified approach different from others, we complete some previous results which are committed to finding sharp conditions for some classical inequalities.

\section{Introduction}

This paper is devoted to studying some convolution inequalities on weighted Lebesgue spaces, for both discrete and continuous versions, with the aim of finding the sharp conditions for boundedness about the Young convolution inequality and the fractional integral operator on these function spaces.

The convolution of two measurable functions on $\mathbb{R}^{n}$ is defined by

$$
(f * g)(x)=\int_{\mathbb{R}^{n}} f(x-y) g(y) d y, x \in \mathbb{R}^{n} .
$$

If $1 \leq p, q, r \leq \infty$ and $1+1 / q=1 / p+1 / r$, then it is well known that the classical Young inequality

$$
\|f * g\|_{L^{q}} \leq\|f\|_{L^{p}}\|g\|_{L^{r}}
$$

plays a fundamental role in studying the convolution operator.

In this paper, we adopt the notation $L^{p} * L^{r} \subset L^{q}$ to denote (1.1), for the sake of simplicity. More generally, for function spaces $X, Y$ and $Z$, the expression of form $X * Y \subset Z$ means that whenever $f \in X, g \in Y$, then $f * g \in Z$ and

$$
\|f * g\|_{Z} \lesssim\|f\|_{X}\|g\|_{Y}
$$

Inequalities of the form (1.2) are usually called the Young-type (convolution) inequalities.

In this paper, we will focus on the Young-type inequalities on the power weighted Lebesgue spaces. Let $s$ be a real number and $1 \leq p \leq \infty$. We use $\mathbb{L}(p, s)$ to denote the weighted $L^{p}$ Lebesgue space with power weight $|x|^{s}$, and $L(p, s)$ to denote the weighted $L^{p}$ Lebesgue space with power weight (without a singularity at the origin) $\langle x\rangle^{s}=\left(1+|x|^{2}\right)^{s / 2}$. Also, the space $l(p, s)$ denotes the discrete counterpart of $L(p, s)$. From a technical point of view, if we ignore the possible singularity of the weight $|x|^{s}$ at the origin as in Proposition [3.2, $l(p, s)$ can be also regarded as the discrete counterpart 
of $\mathbb{L}(p, s)$. In fact, the relationship between $l(p, s)$ and $\mathbb{L}(p, s)$ are quite important for our proof. Since $L(p, 0)=\mathbb{L}(p, 0)=L^{p}$, one easily expects an immediate extension of the classical Young inequality: the inclusion

$$
\mathbb{L}\left(q_{1}, s_{1}\right) * \mathbb{L}\left(q_{2}, s_{2}\right) \subset \mathbb{L}(q, s)
$$

holds for appropriate indices $q_{1}, q_{2}, q$ and $s_{1}, s_{2}, s$. To this end, finding sharp conditions on these indices to ensure the Young inequality

$$
\|f * g\|_{\mathbb{L}(q, s)} \precsim\|f\|_{\mathbb{L}\left(q_{1}, s_{1}\right)}\|g\|_{\mathbb{L}\left(q_{2}, s_{2}\right)}
$$

is considerable and interesting, and this inequality and its varieties might play a pivotal role when we study the convolution operators in the weighted Lebesgue spaces. This problem of course motivated a lot of research works. In the following we briefly review the historical development, by listing a few of research articles related to the topic in this paper.

The study of Young's inequality on the spaces $\mathbb{L}(p, s)$ can be dated back as early as thirty years ago. In 1983, Kerman obtained the following theorem. Theorem A (Kerman [11]). Let $1 \leq q, q_{1}, q_{2} \leq \infty, s, s_{1}, s_{2} \in \mathbb{R}$. Suppose that $(\mathbf{q}, \mathbf{s})=\left(q, q_{1}, q_{2}, s, s_{1}, s_{2}\right)$ satisfies

$$
\left\{\begin{array}{l}
s \leq s_{1}, s \leq s_{2}, 0 \leq s_{1}+s_{2} \\
1+\frac{1}{q}+\frac{s}{n}=\frac{1}{q_{1}}+\frac{s_{1}}{n}+\frac{1}{q_{2}}+\frac{s_{2}}{n}, \frac{1}{q} \leq \frac{1}{q_{1}}+\frac{1}{q_{2}} \\
\frac{1}{q}+\frac{s}{n}<\frac{1}{q_{1}}+\frac{s_{1}}{n}, \frac{1}{q}+\frac{s}{n}<\frac{1}{q_{2}}+\frac{s_{2}}{n}, \frac{1}{q}+\frac{s}{n}>0 \\
q \neq \infty, q_{1}, q_{2} \neq 1 .
\end{array}\right.
$$

Then

$$
\mathbb{L}\left(q_{1}, s_{1}\right) * \mathbb{L}\left(q_{2}, s_{2}\right) \subset \mathbb{L}(q, s) .
$$

The above convolution inequality was also studied by Bui [3], among other authors. Further weighted inequalities for convolutions can be found in [2, 12, 16]. In [3], Bui obtained some necessary conditions for the inclusion (1.3). However, these necessary conditions are not matched the sufficient conditions in Theorem A. Bui thus posed the question for finding the sharp conditions on (1.3). This question was solved just very recently by Nursultanov and Tikhonov [17] in the ranges $1<q_{1}, q_{2}<\infty$ and $1<q \leq \infty$, but with an extra assumption $1 / q \leq 1 / q_{1}+1 / q_{2}$. We note that the result of Nursultanov and Tikhonov does not imply the end point cases $q_{1}, q_{2}=1, \infty$ and $q=1$, while these cases sometime are notably important in applications. Also, the extra condition $1 / q \leq 1 / q_{1}+1 / q_{2}$ seems little odd. Therefore, 
based on these observations, in this paper we will give a complete answer to Bui's question by establishing sharp (sufficient and necessary) conditions of (1.3) in the full ranges $1 \leq q_{1}, q_{2} \leq \infty$ and $1 \leq q \leq \infty$. More significantly, our result removes the extra assumption $1 / q \leq 1 / q_{1}+1 / q_{2}$ (this condition actually, in many cases, is implicitly contained in the necessary conditions).

Since the method used by Nursultanov and Tikhonov is based on an extra assumption and it also raises some difficulties to treat the end point cases, in this paper we will use a quite different approach. We first study the convolution inequalities in the discrete weighted Lebesgue spaces $l(q, s)$. Then we reduce the continuous case to the discrete one to reach our target. On the other hand, we find that the convolution inequalities in the discrete case itself is of interest. We will show that the discrete form of weighted convolution inequality not only has a closed relation to its continuous counterpart, but also is a powerful tool to study the algebraic property of the modulation spaces (see Theorem 1.4).

We also notice that a recent paper [26] also addresses the Young inequality on the spaces $L(p, s)$. The authors establish some sufficient conditions on $L\left(q_{1}, s_{1}\right) * L\left(q_{2}, s_{2}\right) \subset L(q, s)$. They also find some partial necessary conditions. However, there is a big distance between sufficiency and necessity. Again, their methods are different from ours.

As a conclusion, in the full range $1 \leq q, q_{1}, q_{2} \leq \infty$, using different methods from others we will find the sharp conditions for the convolution inequalities in both discrete and continuous weighted Lebesgue spaces.

Let us first list 4 important relations among the indices $q, q_{1}, q_{2}$, and $s, s_{1}, s_{2}$.

$$
\begin{aligned}
& \left(\mathcal{A}_{1}\right)\left\{\begin{array}{l}
s \leq s_{1}, s \leq s_{2}, 0 \leq s_{1}+s_{2}, \\
1+\left(\frac{1}{q}+\frac{s}{n}\right) \vee 0<\left(\frac{1}{q_{1}}+\frac{s_{1}}{n}\right) \vee 0+\left(\frac{1}{q_{2}}+\frac{s_{2}}{n}\right) \vee 0, \\
\frac{1}{q}+\frac{s}{n} \leq \frac{1}{q_{1}}+\frac{s_{1}}{n}, \frac{1}{q}+\frac{s}{n} \leq \frac{1}{q_{2}}+\frac{s_{2}}{n}, 1 \leq \frac{1}{q_{1}}+\frac{s_{1}}{n}+\frac{1}{q_{2}}+\frac{s_{2}}{n}, \\
(q, s)=\left(q_{1}, s_{1}\right) \text { if } \frac{1}{q}+\frac{s}{n}=\frac{1}{q_{1}}+\frac{s_{1}}{n}, \\
(q, s)=\left(q_{2}, s_{2}\right) \text { if } \frac{1}{q}+\frac{s}{n}=\frac{1}{q_{2}}+\frac{s_{2}}{n}, \\
\left(q_{1}^{\prime},-s_{1}\right)=\left(q_{2}, s_{2}\right) \text { if } 1=\frac{1}{q_{1}}+\frac{s_{1}}{n}+\frac{1}{q_{2}}+\frac{s_{2}}{n}
\end{array}\right. \\
& \left(\mathcal{A}_{2}\right)\left\{\begin{array}{l}
s=s_{1}=s_{2}=0 \\
q=q_{1}, q_{2}=1 \text { or } q=q_{2}, q_{1}=1 \text { or } q=\infty, \frac{1}{q_{1}}+\frac{1}{q_{2}}=1
\end{array}\right.
\end{aligned}
$$

$$
\left(\mathcal{A}_{3}\right)\left\{\begin{array}{l}
s \leq s_{1}, s \leq s_{2}, \\
\frac{1}{q_{1}}+\frac{1}{q_{2}}=1, s_{1}+s_{2}=0, \\
\frac{1}{q}+\frac{s}{n}<0 \leq \frac{1}{q_{1}}+\frac{s_{1}}{n}, \frac{1}{q_{2}}+\frac{s_{2}}{n} ;
\end{array}\right.
$$




$$
\left(\mathcal{A}_{4}\right)\left\{\begin{array}{l}
s \leq s_{1}, s \leq s_{2}, 0 \leq s_{1}+s_{2} \\
1+\frac{1}{q}+\frac{s}{n}=\frac{1}{q_{1}}+\frac{s_{1}}{n}+\frac{1}{q_{2}}+\frac{s_{2}}{n}, \frac{1}{q} \leq \frac{1}{q_{1}}+\frac{1}{q_{2}} \\
\frac{1}{q}+\frac{s}{n}<\frac{1}{q_{1}}+\frac{s_{1}}{n}, \frac{1}{q}+\frac{s}{n}<\frac{1}{q_{2}}+\frac{s_{2}}{n}, \frac{1}{q}+\frac{s}{n}>0 \\
q \neq \infty, q_{1}, q_{2} \neq 1, \text { if } s=s_{1} \text { or } s=s_{2} .
\end{array}\right.
$$

Throughout this paper, we use $p^{\prime}$ to denote the dual index of $p$ such that $1 / p+1 / p^{\prime}=1$, and use the notation

$$
a \vee b=\max \{a, b\}
$$

Now, we state our main results associated with convolution inequalities on weighted Lebesgue spaces.

Theorem 1.1 (Young's inequality, discrete form, weight $\langle k\rangle$ ). Suppose $1 \leq$ $q, q_{1}, q_{2} \leq \infty, s, s_{1}, s_{2} \in \mathbb{R}$. Then

$$
l\left(q_{1}, s_{1}\right) * l\left(q_{2}, s_{2}\right) \subset l(q, s)
$$

if and only if $(\mathbf{q}, \mathbf{s})=\left(q, q_{1}, q_{2}, s, s_{1}, s_{2}\right)$ satisfies one of the conditions $\mathcal{A}_{i}$, $i=1,2,3,4$.

Theorem 1.2 (Young's inequality, continuous, weight $|x|)$. Suppose $1 \leq$ $q, q_{1}, q_{2} \leq \infty, s, s_{1}, s_{2} \in \mathbb{R}$. Then

$$
\mathbb{L}\left(q_{1}, s_{1}\right) * \mathbb{L}\left(q_{2}, s_{2}\right) \subset \mathbb{L}(q, s)
$$

if and only if $(\mathbf{q}, \mathbf{s})$ satisfies one of conditions $\mathcal{A}_{2}$ and $\mathcal{A}_{4}$.

Theorem 1.3 (Young's inequality, continuous, weight $\langle x\rangle$ ). Suppose $1 \leq$ $q, q_{1}, q_{2} \leq \infty, s, s_{1}, s_{2} \in \mathbb{R}$. Then

$$
L\left(q_{1}, s_{1}\right) * L\left(q_{2}, s_{2}\right) \subset L(q, s)
$$

if and only if $(\mathbf{q}, \mathbf{s})$ satisfies

$$
1+\frac{1}{q} \geq \frac{1}{q_{1}}+\frac{1}{q_{2}}
$$

and one of the conditions $\mathcal{A}_{i}, i=1,2,3,4$.

As an application, we will study the product inequalities on the modulation spaces. Then, as a consequence, we obtain an algebraic property for modulation spaces, while it is known that this algebraic property is a key 
issue to study certain nonlinear Cauchy problem of dissipative partial differential equations on the modulation spaces [28]. The modulation space $M_{p, q}^{s}$ was introduced by Feichtinger [7] in 1983 by means of the short-time Fourier transform. Another equivalent definition of $M_{p, q}^{s}$ can be given by applying the frequency-uniform localizations (see [28] for details). The interested reader may find a lot of research articles, in the literature, that address the space $M_{p, q}^{s}$, as well as its many applications. For instance, see [29] for some basic properties of modulation spaces, [1, 8] for the study of boundedness on modulation spaces for certain operators. Particularly, it is known that the modulation space serves as a good alternative working frame, in many cases, in the study of partial differential equations, see [10, 28, 19]. The definitions of modulation space will be presented in Section 2, but we would like to give the reader an earlier notice that Theorem 1.1 is a crucial inequality to obtain the product inequalities on modulation spaces. More precisely, using Theorem 1.1 we will establish the following algebraic property of the modulation spaces.

Theorem 1.4 (Product on modulation spaces). Suppose $1 \leq p, p_{1}, p_{2} \leq \infty$, $1 \leq q, q_{1}, q_{2} \leq \infty, s, s_{1}, s_{2} \in \mathbb{R}$. Then

$$
\|f g\|_{M_{p, q}^{s}} \lesssim\|f\|_{M_{p_{1}, q_{1}}^{s_{1}}}\|g\|_{M_{p_{2}, q_{2}}^{s_{2}}}
$$

holds for all $f, g \in \mathscr{S}$ if and only if $1 / p \leq 1 / p_{1}+1 / p_{2}$ and (q, $\left.\mathbf{s}\right)$ satisfies one of the conditions $\mathcal{A}_{i}, i=1,2,3,4$.

We note that a simpler case of the above theorem was obtained in [4]. However, using our method we are able to study a more general bilinear Fourier multiplier $T(f, g)$ that takes the product $f g$ as a special case. The bilinear Fourier multiplier $T$ with symbol $m(\xi, \eta)$ is defined on the product Schwartz space $\mathscr{S} \times \mathscr{S}$ by

$$
T(f, g)(x)=\int_{\mathbb{R}^{n}} \int_{\mathbb{R}^{n}} m(\xi, \eta) \widehat{f}(\xi) \widehat{g}(\eta) e^{2 \pi i<x, \xi+\eta>} d \xi d \eta
$$

for all $f, g \in \mathscr{S}$. With the relation of the Fourier transform and its inverse, it is easy to see that

$$
T(f, g)(x)=f(x) g(x)
$$

if $m(\xi, \eta) \equiv 1$.

An interesting question is whether $T(f, g)$ is bounded on the modulation spaces provided it is bounded on certain Lebesgue spaces. We find the sharp conditions to answer this question. 
Theorem 1.5 (Multi-linear Fourier multipliers on modulation spaces). Suppose $1 \leq q, q_{1}, q_{2} \leq \infty, s, s_{1}, s_{2} \in \mathbb{R}$. Then

$$
\|T(f, g)\|_{L^{p}} \lesssim\|f\|_{L^{p_{1}}}\|g\|_{L^{p_{2}}} \Longrightarrow\|T(f, g)\|_{M_{p, q}^{s}} \lesssim\|f\|_{M_{p_{1}, q_{1}}^{s_{1}}}\|g\|_{M_{p_{2}, q_{2}}^{s_{2}}}
$$

holds for any bilinear Fourier multiplier $T$ and $1 \leq p, p_{1}, p_{2} \leq \infty$, if and only if $(\mathbf{q}, \mathbf{s})$ satisfies one of the conditions $\mathcal{A}_{i}, i=1,2,3,4$.

The convolution $f * g$ may be naturally regarded as a bilinear operator. One may fix the function $g$ as the kernel function and consider the operator

$$
T_{g} f(x)=(f * g)(x)
$$

In Fourier analysis, an important operator with this form is the fractional integral operator (or Riesz potential) $I_{\lambda}$ defined by

$$
\left(I_{\lambda} f\right)(x)=\int_{\mathbb{R}^{n}} \frac{f(y)}{|x-y|^{n-\lambda}} d y, \quad 0<\lambda<n .
$$

The famous Hardy-Littlewood-Sobolev theorem gives the boundedness of $I_{\lambda}$ from $L^{p}\left(\mathbb{R}^{n}\right)$ to $L^{q}\left(\mathbb{R}^{n}\right)$, provided $1<p \leq q<\infty$ and $1 / q=1 / p-$ $\lambda / n$. Continuity properties of the potential operator in the Lebesgue spaces are well known, see [24, 9]. The following weighted version of the HardyLittlewood-Sobolev theorem was obtained by Stein and Weiss five decades ago in 23 .

Theorem B (Stein-Weiss [23]). Suppose $1<p \leq q<\infty, s, t \in \mathbb{R}$. If

$$
\left\{\begin{array}{l}
s \leq t \\
\frac{1}{q}+\frac{s}{n}=\frac{1}{p}+\frac{t}{n}-\frac{\lambda}{n} \\
\frac{1}{p}+\frac{t}{n}<1, \frac{1}{q}+\frac{s}{n}>0
\end{array}\right.
$$

then

$$
I_{\lambda}: \mathbb{L}(p, t) \rightarrow \mathbb{L}(q, s) .
$$

A more general result on $I_{\lambda}: \mathbb{L}(p, t) \rightarrow \mathbb{L}(q, s)$, including the endpoint $p=1$ or $q=\infty$ can be found in [25], in which the author provided an alternative proof. Under the assumption $1 \leq p \leq q<\infty$, Duoandikoetxea [6] found some necessity conditions on the map $I_{\lambda}: \mathbb{L}(p, t) \rightarrow \mathbb{L}(q, s)$. Also in the same paper, Duoandikoetxea obtained the (partial) necessary conditions in the radial case. Recently, Nowak and Stempak claimed the complete result in the radial case, by finding the sharp conditions for $I_{\lambda}: \mathbb{L}(p, t) \rightarrow$ $\mathbb{L}(q, s)$, including the endpoint $q=\infty$ (see Corollary 2.6 in [15]). Some work associated with the weighted inequality for fractional integral operator can 
be found in [5, 13, 14, 18, 20, 21]. On the other hand, as mentioned in Stein-Wainger 22], the discrete analogue of the fractional integral operator is given by

$$
\left(\mathcal{I}_{\lambda} f\right)(k)=\sum_{j \in \mathbb{Z}^{n}, j \neq k} \frac{f(j)}{|k-j|^{n-\lambda}} .
$$

As the second application of our main results on Young-type inequalities, we will study the fractional integral operator in both discrete and continuous case. Our results and methods allow us to obtain the sharp conditions for the boundedeness of fractional integral operator on weighted Lebesgue spaces. Especially, in the continuous case, we optimize some previous results by finding the sharp conditions for the boundedness of $L^{p}-L^{q}$ estimates of fractional integral operators with power weights. Our proof mainly depends on the discretization of the operator, which is quite different from the methods used by other authors.

Now, we list our main results associated with fractional integral operators.

Theorem 1.6 (Fractional integral operator, discrete form, weight $\langle k\rangle$ ). Suppose $1 \leq p, q \leq \infty, t, s \in \mathbb{R}$. Then

$$
\mathcal{I}_{\lambda}: l(p, t) \rightarrow l(q, s)
$$

if and only if $(q, p, s, t)$ satisfies one of the following conditions

$$
\begin{aligned}
& \left(\mathcal{C}_{1}\right)\left\{\begin{array}{l}
s \leq t, \\
\frac{\lambda}{n}+\left(\frac{1}{q}+\frac{s}{n}\right) \vee 0<\left(\frac{1}{p}+\frac{t}{n}\right) \vee 0 \\
\frac{\lambda}{n}+\frac{1}{q}+\frac{s}{n} \leq 1, \\
\left(q^{\prime},-s\right)=(1, \lambda-n) \text { if } \frac{\lambda}{n}+\frac{1}{q}+\frac{s}{n}=1
\end{array}\right. \\
& \left(\mathcal{C}_{3}\right)\left\{\begin{array}{l}
s \leq t, \\
p=1, t=\lambda-n, \frac{1}{q}+\frac{s}{n}<0
\end{array}\right. \\
& \left(\mathcal{C}_{4}\right)\left\{\begin{array}{l}
s \leq t, \\
\frac{\lambda}{n}+\frac{1}{q}+\frac{s}{n}=\frac{1}{p}+\frac{t}{n}, \frac{1}{q} \leq \frac{1}{p} \\
\frac{1}{q}+\frac{s}{n}>0, \frac{1}{p}+\frac{t}{n}<1, \\
q \neq \infty, p \neq 1, \text { if } s=t
\end{array}\right.
\end{aligned}
$$

Theorem 1.7 (Fractional integral operator, continuous form, weight $|x|$ ). Let $1 \leq p, q \leq \infty, t, s \in \mathbb{R}$. Then

$$
I_{\lambda}: \mathbb{L}(p, t) \rightarrow \mathbb{L}(q, s)
$$

if and only if $(q, p, s, t)$ satisfies $\mathcal{C}_{4}$. 
In Theorem 1.6, to maintain the unified format of proof as in Theorem 1.1, we skip $\mathcal{C}_{2}$ to name the conditions. In fact, in our unified method, the conditions $\mathcal{C}_{i}$ and $\mathcal{A}_{i}$ are correspondence for each $\mathrm{i}=1,2,3,4$. The subscript $i=2$ means the double endpoint cases which can be proved to be trivial. Under this method of classification, we actually have $\mathcal{C}_{2}=\emptyset$. One can also see the proof of Theorem 1.6 in this direction.

In addition, the subscript $i=1$ means the case in which we can use embedding argument to reduce the proof to a more standard case (see Proposition 3.1), the case $i=3$ is actually the dual of the case $i=1$. Finally, condition for $i=4$ collect the cases which is closely linked to the continuous form.

We remark that each of Theorems 1.1 to 1.7 can be verified independently. For convenience, we sometimes use one Theorem 1.1 to prove other theorems for an easy approach. Our methods in this paper is in the spirit of discretization, even in the process of dealing with the continuous case. This is totally different from the methods used in the other references about this topic.

We also remark that the nonnegative functions (or sequences) are enough for most of the proofs in this paper. So, if there is no special explanation, the functions we use in the proofs should be presumed nonnegative.

\section{Preliminaries and Definitions}

Let $C$ be a positive constant that may depend on $n, p_{i}, q_{i}, s_{i}, t, \lambda(i=1,2)$. The notation $X \lesssim Y$ denotes the statement that $X \leq C Y$, the notation $X \sim Y$ means the statement $X \lesssim Y \lesssim X$, and the notation $X \simeq Y$ denotes the statement $X=C Y$. For a multi-index $k=\left(k_{1}, k_{2}, \ldots, k_{n}\right) \in \mathbb{Z}^{n}$, we denote $|k|_{\infty}:=\sup _{i=1,2, \ldots, n}\left|k_{i}\right|$, and $\langle k\rangle:=\left(1+|k|^{2}\right)^{1 / 2}$.

Let $\mathscr{S}:=\mathscr{S}\left(\mathbb{R}^{n}\right)$ be the Schwartz space and $\mathscr{S}^{\prime}:=\mathscr{S}^{\prime}\left(\mathbb{R}^{n}\right)$ be the space of tempered distributions. We define the Fourier transform $\mathcal{F} f$ and the inverse Fourier $\mathcal{F}^{-1} f$ of $f \in \mathscr{S}\left(\mathbb{R}^{n}\right)$ by

$\mathcal{F} f(\xi)=\hat{f}(\xi)=\int_{\mathbb{R}^{n}} f(x) e^{-2 \pi i x \cdot \xi} d x, \quad \mathcal{F}^{-1} f(x)=\hat{f}(-x)=\int_{\mathbb{R}^{n}} f(\xi) e^{2 \pi i x \cdot \xi} d \xi$

We recall, in the following, the definitions and some properties of the function spaces involved in this paper.

Definition 2.1. Let $s \in \mathbb{R}, 0<p, q \leq \infty$. The function space $\mathbb{L}_{x}(p, s)$ 
consists of all measurable functions $f$ such that

$$
\|f\|_{\mathbb{L}_{x}(p, s)}= \begin{cases}\left(\int_{\mathbb{R}^{n}}|f(x)|^{p}|x|^{p s} d x\right)^{1 / p}, & p<\infty \\ e s s \sup _{x \in \mathbb{R}^{n}}|f(x)||x|^{s}, & p=\infty\end{cases}
$$

is finite. The function space $L_{x}(p, s)$ consists of measurable functions $f$ such that

$$
\|f\|_{L_{x}(p, s)}= \begin{cases}\left(\int_{\mathbb{R}^{n}}|f(x)|^{p}\langle x\rangle^{p s} d x\right)^{1 / p}, & p<\infty \\ e s s \sup _{x \in \mathbb{R}^{n}}|f(x)|\langle x\rangle^{s}, & p=\infty\end{cases}
$$

is finite. If $f$ is defined on $\mathbb{Z}^{n}$, we denote its $l_{k}(p, s)$ norm

$$
\|f\|_{l_{k}(p, s)}= \begin{cases}\left(\sum_{k \in \mathbb{Z}^{n}}|f(k)|^{p}\langle k\rangle^{p s}\right)^{1 / p}, & p<\infty \\ \sup _{k \in \mathbb{Z}^{n}}|f(k)|\langle k\rangle^{s}, & p=\infty\end{cases}
$$

and let $l_{k}(p, s)$ be the (quasi-)Banach space of functions $f: \mathbb{Z}^{n} \rightarrow \mathbb{C}$ whose $l_{k}(p, s)$ norm is finite. We write $\mathbb{L}(p, s), L(p, s), l(p, s)$ for short respectively, if there is no confusion. We also denote $L^{p}=\mathbb{L}(p, 0)=L(p, 0), l^{p}=l(p, 0)$ for short.

To introduce the modulation space, we first give the definition of the short-time Fourier transform. For a fixed nonzero $\phi \in \mathscr{S}$, the short-time Fourier transform of $f \in \mathscr{S}$ with respect to the window function $\phi$ is given by

$$
V_{\phi} f(x, \xi)=\int_{\mathbb{R}^{n}} f(y) \overline{\phi(y-x)} e^{-2 \pi i y \cdot \xi} d y .
$$

The norm on modulation space is given by

$$
\begin{aligned}
\|f\|_{M_{p, q}^{s}} & =\|\| V_{\phi} f(x, \xi)\left\|_{L_{x}^{p}}\right\|_{L_{\xi}(q, s)} \\
& =\left(\int_{\mathbb{R}^{n}}\left(\int_{\mathbb{R}^{n}}\left|V_{\phi} f(x, \xi)\right|^{p} d x\right)^{q / p}\langle\xi\rangle^{s q} d \xi\right)^{1 / q},
\end{aligned}
$$

with a natural modification for $p=\infty$ or $q=\infty$. Note that this definition is independent of the choice of the window function.

Applying the frequency-uniform localization techniques, one can give an alternative definition of modulation spaces (see [28] for details). For $k \in \mathbb{Z}^{n}$, we denote by $Q_{k}$ the unit cube centered at $k$. The family $\left\{Q_{k}\right\}_{k \in \mathbb{Z}^{n}}$ 
constitutes a decomposition of $\mathbb{R}^{n}$. Let $\rho \in \mathscr{S}\left(\mathbb{R}^{n}\right), \rho: \mathbb{R}^{n} \rightarrow[0,1]$ be a smooth function satisfying $\rho(\xi)=1$ for $|\xi|_{\infty} \leq 1 / 2$ and $\rho(\xi)=0$ for $|\xi| \geq 3 / 4$. Let $\rho_{k}$ be a translation of $\rho$,

$$
\rho_{k}(\xi)=\rho(\xi-k), k \in \mathbb{Z}^{n} .
$$

Since $\rho_{k}(\xi)=1$ for $\xi \in Q_{k}$, we have that $\sum_{k \in \mathbb{Z}^{n}} \rho_{k}(\xi) \geq 1$ for all $\xi \in \mathbb{R}^{n}$. Denote

$$
\sigma_{k}(\xi)=\rho_{k}(\xi)\left(\sum_{l \in \mathbb{Z}^{n}} \rho_{l}(\xi)\right)^{-1}, \quad k \in \mathbb{Z}^{n} .
$$

Then $\left\{\sigma_{k}(\xi)\right\}_{k \in \mathbb{Z}^{n}}$ constitutes a smooth decomposition of $\mathbb{R}^{n}$, where $\sigma_{k}(\xi)=$ $\sigma(\xi-k)$. The frequency-uniform decomposition operators are defined by

$$
\square_{k}:=\mathscr{F}^{-1} \sigma_{k} \mathscr{F}
$$

for $k \in \mathbb{Z}^{n}$. With the family $\left\{\square_{k}\right\}_{k \in \mathbb{Z}^{n}}$, an alternative norm of modulation space can be defined by

$$
\left(\sum_{k \in \mathbb{Z}^{n}}\langle k\rangle^{s q}\left\|\square_{k} f\right\|_{p}^{q}\right)^{1 / q}
$$

with a natural modification for $p=\infty$ or $q=\infty$. We recall that this definition is independent of the choice of $\sigma$ and that this norm is equivalent to the norm defined in (2.1) (see [28]). So we use the same symbol $\|f\|_{M_{p, q}^{s}}$ to denote these two modulation space norms.

Lemma 2.2 (Embedding of $L^{p}$ with Fourier compact support, [27]). Let $0<p_{1} \leq p_{2} \leq \infty$ and assume supp $\hat{f} \subseteq B\left(x_{0}, R\right)$ for some $x_{0} \in \mathbb{R}^{n}, R>0$. We have

$$
\|f\|_{L^{p_{2}}} \leq C R^{n\left(\frac{1}{p_{1}}-\frac{1}{p_{2}}\right)}\|f\|_{L^{p_{1}}}
$$

where $C$ is independent of $f$ and $x_{0}$.

Next, we list some propositions used in the proof of our main theorems. These propositions are not difficult to be verified, so we only give partial proof details and give some hints.

Proposition 2.3 (Sharpness of embedding, discrete form). Suppose $0<$ $q, q_{1}, q_{2} \leq \infty, s, s_{1}, s_{2} \in \mathbb{R}$. Then

$$
l\left(q_{1}, s_{1}\right) \subset l\left(q_{2}, s_{2}\right)
$$

holds if and only if

$$
\left\{\begin{array} { l } 
{ s _ { 2 } \leq s _ { 1 } } \\
{ \frac { 1 } { q _ { 2 } } + \frac { s _ { 2 } } { n } < \frac { 1 } { q _ { 1 } } + \frac { s _ { 1 } } { n } }
\end{array} \quad \text { or } \quad \left\{\begin{array}{l}
s_{2}=s_{1} \\
q_{2}=q_{1} .
\end{array}\right.\right.
$$


Proof. The sufficiency can be verified by the Hölder inequality and the fact that $l_{q_{1}} \subset l_{q_{2}}$ for $1 / q_{2} \leqslant 1 / q_{1}$.

To prove the necessity, we firstly obtain $\frac{1}{q_{2}}+\frac{s_{2}}{n} \leqslant \frac{1}{q_{1}}+\frac{s_{1}}{n}$ by the same method as in the proof of (3.2). For a fixed $N \in \mathbb{N}$, we take $a_{k, N}=1$ if $k=N$ and $a_{k, N}=1$ if $k=N$, then $s_{2} \leqslant s_{1}$ follows by

$$
\langle N\rangle^{s_{2}} \sim\left\|\left\{a_{k, N}\right\}\right\|_{l\left(q_{2}, s_{2}\right)} \lesssim\left\|\left\{a_{k, N}\right\}\right\|_{l\left(q_{1}, s_{1}\right)} \sim\langle N\rangle^{s_{1}}
$$

as $|N| \rightarrow \infty$.

Especially, for $\frac{1}{q_{2}}+\frac{s_{2}}{n}=\frac{1}{q_{1}}+\frac{s_{1}}{n}$, we take

$$
b_{k, N}= \begin{cases}\langle k\rangle^{-n\left(1 / q_{1}+s_{1} / n\right)}, & \text { for }|k| \leq N \\ 0, & \text { otherwise }\end{cases}
$$

Observing that

$$
(\ln N)^{1 / q_{2}} \sim\left\|\left\{b_{k, N}\right\}\right\|_{l\left(q_{2}, s_{2}\right)} \lesssim\left\|\left\{b_{k, N}\right\}\right\|_{l\left(q_{1}, s_{1}\right)} \sim(\ln N)^{1 / q_{1}}
$$

we deduce $1 / q_{2} \leqslant 1 / q_{1}$ by letting $|N| \rightarrow \infty$. Recalling $\frac{1}{q_{2}}+\frac{s_{2}}{n}=\frac{1}{q_{1}}+\frac{s_{1}}{n}$ and $s_{2} \leqslant s_{1}$, we actually have $s_{2}=s_{1}$ and $q_{2}=q_{1}$ in this case.

In this paper, the space $l(q, s)$ is quite important in our proof.

Proposition 2.4 (Sharpness of embedding, continuous form). Suppose $0<$ $q, q_{1}, q_{2} \leq \infty, s, s_{1}, s_{2} \in \mathbb{R}$. Then

$$
L\left(q_{1}, s_{1}\right) \subset L\left(q_{2}, s_{2}\right)
$$

holds if and only if

$$
\left\{\begin{array} { l } 
{ s _ { 2 } \leq s _ { 1 } } \\
{ \frac { 1 } { q _ { 2 } } \geq \frac { 1 } { q _ { 1 } } } \\
{ \frac { 1 } { q _ { 2 } } + \frac { s _ { 2 } } { n } < \frac { 1 } { q _ { 1 } } + \frac { s _ { 1 } } { n } }
\end{array} \quad \text { or } \quad \left\{\begin{array}{l}
s_{2}=s_{1} \\
q_{2}=q_{1}
\end{array}\right.\right.
$$

Proof. The sufficiency can be verified by Hölder's inequality. In the necessity part, we take $f(x)=\chi_{B(0, a)}$ for $a \in(0,1)$. Then $1 / q_{2} \geqslant 1 / q_{1}$ follows by letting $a \rightarrow 0$ in

$$
a^{n / q_{2}} \sim\|f\|_{L\left(q_{2}, s_{2}\right)} \lesssim\|f\|_{L\left(q_{1}, s_{1}\right)} \sim a^{n / q_{1}}
$$

The rest of the proof is similarly as that in Proposition 2.3. 
Proposition 2.5 (Young's inequality, discrete form). Suppose $0<q, q_{1}, q_{2} \leq$ $\infty$. Then

$$
l^{q_{1}} * l^{q_{2}} \subset l^{q}
$$

holds if and only if

$$
\left\{\begin{array}{l}
1+\frac{1}{q} \leq \frac{1}{q_{1}}+\frac{1}{q_{2}} \\
\frac{1}{q} \leq \frac{1}{q_{1}}, \frac{1}{q} \leq \frac{1}{q_{2}}
\end{array}\right.
$$

Proof. Since the necessity part can be verified by the same method as in the proof of Theorem 1.1, we only give the proof for the sufficiency. If $q \leqslant 1$, we have $l^{q} * l^{q} \subset l^{q}$, then the sufficiency can be verified by

$$
l^{q_{1}} * l^{q_{2}} \subset l^{q} * l^{q} \subset l^{q}
$$

where we use $l^{q_{1}} \subset l^{q}, l^{q_{2}} \subset l^{q}$ by the fact $1 / q \leqslant 1 / q_{1}, 1 / q_{2}$.

If $q \geqslant 1, q_{1} \geqslant 1, q_{2} \leqslant 1$, we deduce

$$
l^{q_{1}} * l^{q_{2}} \subset l^{q} * l^{1} \subset l^{q}
$$

where we use $l^{q_{2}} \subset l^{1}$ in this case. By the symmetry, the case $q \geqslant 1, q_{2} \geqslant 1$, $q_{1} \leqslant 1$ can be handled by the same way.

If $q \geqslant 1, q_{1} \geqslant 1, q_{2} \geqslant 1$, we can choose $r_{j} \geqslant 1(j=1,2)$ such that

$$
1+1 / q=1 / r_{1}+1 / r_{2}, r_{j} \geqslant q_{j}(j=1,2)
$$

Using Young's inequality, we obtain $l^{r_{1}} * l^{r_{2}} \subset l^{q}$. It implies that

$$
l^{q_{1}} * l^{q_{2}} \subset l^{r_{1}} * l^{r_{2}} \subset l^{q}
$$

by using $l^{q_{i}} \subset l^{r_{i}}(i=1,2)$ in this case.

Proposition 2.6 (Young's inequality, continuous form). Let $0<q, q_{1}, q_{2} \leq$ $\infty$. Then

$$
L^{q_{1}} * L^{q_{2}} \subset L^{q}
$$

holds if and only if

$$
\left\{\begin{array}{l}
1+\frac{1}{q}=\frac{1}{q_{1}}+\frac{1}{q_{2}} \\
\frac{1}{q} \leq \frac{1}{q_{1}}, \frac{1}{q} \leq \frac{1}{q_{2}} .
\end{array}\right.
$$

Proof. The necessity can be verified by the same method as in the proof of Theorem 1.2, Observing the conditions is equivalent to $1 / q=1 / q_{1}+1 / q_{2}$, $q, q_{1}, q_{2} \geqslant 1$, the sufficiency follows by the classical Young's inequality. 
Proposition 2.7 (Integral capability of weight $\langle k\rangle$ ). Suppose $s>0, s \leq s_{1}$, $s \leq s_{2}$. Then

$$
\begin{gathered}
\left\{t \in(0, \infty]:\left\|\frac{\langle k\rangle^{s}}{\langle k-j\rangle^{s_{1}}\langle j\rangle^{s_{2}}}\right\|_{l_{j}^{t}} \lesssim 1, \text { for all } k \in \mathbb{Z}^{n}\right\} \\
=\left\{\begin{array}{l}
\left(\frac{n}{s_{1}+s_{2}-s}, \infty\right], \text { if } s=s_{1} \text { or } s=s_{2}, \\
{\left[\frac{n}{s_{1}+s_{2}-s}, \infty\right], \text { if } s<s_{1} \text { and } s<s_{2} .}
\end{array}\right.
\end{gathered}
$$

Proof. By a direct calculation,

$$
\begin{aligned}
& \sum_{j \in \mathbb{Z}^{n}} \frac{\langle k\rangle^{s t}}{\langle k-j\rangle^{s_{1} t}\langle j\rangle^{s_{2} t}} \\
= & \sum_{|j| \leq|k| / 2}+\sum_{|j-k| \leq|k| / 2}+\sum_{|j| \geq 2 k}+\sum_{\text {others }} \\
\sim & \sum_{|j| \leq|k| / 2} \frac{\langle k\rangle^{\left(s-s_{1}\right) t}}{\langle j\rangle^{s_{2} t}}+\sum_{|j| \leq|k| / 2} \frac{\langle k\rangle^{\left(s-s_{2}\right) t}}{\langle j\rangle^{s_{1} t}}+\sum_{|j| \geq 2|k|} \frac{\langle k\rangle^{s t}}{\langle j\rangle^{\left(s_{1}+s_{2}\right) t}}+\langle k\rangle^{n+\left(s-s_{1}-s_{2}\right) t} \\
= & : I+I I+I I I+I V .
\end{aligned}
$$

One can verify that $I I I$ and $I V$ have uniform bounds on $k$ if and only if $t \geq$ $n /\left(s_{1}+s_{2}-s\right)$. On the other hand, under the condition $t \geq n /\left(s_{1}+s_{2}-s\right)$, the term $I$ also has uniform bounds on $k$, unless $t=n / s_{2}$ and $s=s_{1}$. Similarly, one can verify that $I I$ also has uniform bounds on $k$ unless $t=$ $n / s_{1}$ and $s=s_{2}$.

The following two propositions can be verified by the similar technique as in the proof of Proposition 2.7, so we omit their proofs.

Proposition 2.8 (Integral capability of weight $\langle x\rangle$ ). Suppose $s>0, s \leq s_{1}$, $s \leq s_{2}$. Then

$$
\begin{gathered}
\left\{t:\left\|\frac{\langle x\rangle^{s}}{\langle x-y\rangle^{s_{1}}\langle y\rangle^{s_{2}}}\right\|_{L_{y}^{t}} \lesssim 1, \text { for all } x \in \mathbb{R}^{n}\right\} \\
=\left\{\begin{array}{l}
\left(\frac{n}{s_{1}+s_{2}-s}, \infty\right], \text { if } s=s_{1} \text { or } s=s_{2}, \\
{\left[\frac{n}{s_{1}+s_{2}-s}, \infty\right], \text { if } s<s_{1} \text { and } s<s_{2} .}
\end{array}\right.
\end{gathered}
$$

Proposition 2.9 (Integral capability of weight $|x|$ ). Suppose $s>0, s<s_{1}$, $s<s_{2}$. Then

$$
\left\|\frac{|x|^{s}}{|x-y|^{s_{1}}|y|^{s_{2}}}\right\|_{L_{y}^{\frac{n}{s_{1}+s_{2}-s}}} \lesssim 1
$$

for all $x \in \mathbb{R}^{n}$. 


\section{Discrete weighted Young's inequality - Proof of Theorem 1.1}

\subsection{Notations and procedure of the proof}

We start this section by defining the set

$$
A=\left\{(\mathbf{q}, \mathbf{s}) \in[1, \infty]^{3} \times \mathbb{R}^{3}: l\left(q_{1}, s_{1}\right) * l\left(q_{2}, s_{2}\right) \subset l(q, s)\right\}
$$

We now describe the strategy to characterize the set $A$.

Use $A_{i}$ to denote the set of all $(\mathbf{q}, \mathbf{s}) \in[1, \infty]^{3} \times \mathbb{R}^{3}$ satisfying condition $\mathcal{A}_{i}$, respectively, for $i=1,2,3,4, X$ to denote the set of all $(\mathbf{q}, \mathbf{s}) \in[1, \infty]^{3} \times \mathbb{R}^{3}$ satisfying

$$
\left\{\begin{array}{l}
s \leq s_{1}, s \leq s_{2}, 0 \leq s_{1}+s_{2} \\
1+\left(\frac{1}{q}+\frac{s}{n}\right) \vee 0 \leq\left(\frac{1}{q_{1}}+\frac{s_{1}}{n}\right) \vee 0+\left(\frac{1}{q_{2}}+\frac{s_{2}}{n}\right) \vee 0 \\
\frac{1}{q}+\frac{s}{n} \leq \frac{1}{q_{1}}+\frac{s_{1}}{n}, \frac{1}{q}+\frac{s}{n} \leq \frac{1}{q_{2}}+\frac{s_{2}}{n}, 1 \leq \frac{1}{q_{1}}+\frac{s_{1}}{n}+\frac{1}{q_{2}}+\frac{s_{2}}{n} \\
(q, s)=\left(q_{1}, s_{1}\right), \text { if } \frac{1}{q}+\frac{s}{n}=\frac{1}{q_{1}}+\frac{s_{1}}{n} \\
(q, s)=\left(q_{2}, s_{2}\right), \text { if } \frac{1}{q}+\frac{s}{n}=\frac{1}{q_{2}}+\frac{s_{2}}{n} \\
\left(q_{1}^{\prime},-s_{1}\right)=\left(q_{2}, s_{2}\right), \text { if } 1=\frac{1}{q_{1}}+\frac{s_{1}}{n}+\frac{1}{q_{2}}+\frac{s_{2}}{n}
\end{array}\right.
$$

We also use $X_{1}$ to denote the set of all pairs $(\mathbf{q}, \mathbf{s}) \in X$ satisfying

$$
1+\left(\frac{1}{q}+\frac{s}{n}\right) \vee 0<\left(\frac{1}{q_{1}}+\frac{s_{1}}{n}\right) \vee 0+\left(\frac{1}{q_{2}}+\frac{s_{2}}{n}\right) \vee 0
$$

By this notation, one easily checks that $X_{1}=A_{1}$. We use $X_{2}$ to denote the set of all $(\mathbf{q}, \mathbf{s}) \in X \backslash X_{1}$ satisfying

$$
\frac{1}{q}+\frac{s}{n}=\frac{1}{q_{1}}+\frac{s_{1}}{n} \text { or } \frac{1}{q}+\frac{s}{n}=\frac{1}{q_{2}}+\frac{s_{2}}{n} \text { or } \frac{1}{q}+\frac{s}{n}=0 .
$$

We use $X_{3}$ to denote the set of all $(\mathbf{q}, \mathbf{s}) \in X \backslash\left(X_{1} \cup X_{2}\right)$ satisfying

$$
\frac{1}{q}+\frac{s}{n}<0
$$

Use $X_{4}$ to denote the set of all $(\mathbf{q}, \mathbf{s}) \in X \backslash\left(X_{1} \cup X_{2}\right)$ satisfying

$$
\frac{1}{q}+\frac{s}{n}>0 .
$$


By the above definition, we also obtain that $X_{3}$ is the set of all $(\mathbf{q}, \mathbf{s}) \in$ $[1, \infty]^{3} \times \mathbb{R}^{3}$ satisfying

$$
\left\{\begin{array}{l}
s \leq s_{1}, s \leq s_{2}, 0 \leq s_{1}+s_{2}, \\
1+\left(\frac{1}{q}+\frac{s}{n}\right) \vee 0=\left(\frac{1}{q_{1}}+\frac{s_{1}}{n}\right) \vee 0+\left(\frac{1}{q_{2}}+\frac{s_{2}}{n}\right) \vee 0, \\
\frac{1}{q}+\frac{s}{n}<\frac{1}{q_{1}}+\frac{s_{1}}{n}, \frac{1}{q}+\frac{s}{n}<\frac{1}{q_{2}}+\frac{s_{2}}{n}, 1 \leq \frac{1}{q_{1}}+\frac{s_{1}}{n}+\frac{1}{q_{2}}+\frac{s_{2}}{n}, \frac{1}{q}+\frac{s}{n}<0, \\
\left(q_{1}^{\prime},-s_{1}\right)=\left(q_{2}, s_{2}\right) \text { if } 1=\frac{1}{q_{1}}+\frac{s_{1}}{n}+\frac{1}{q_{2}}+\frac{s_{2}}{n} ;
\end{array}\right.
$$

$X_{4}$ is the set of all $(\mathbf{q}, \mathbf{s}) \in[1, \infty]^{3} \times \mathbb{R}^{3}$ satisfying

$$
\left\{\begin{array}{l}
s \leq s_{1}, s \leq s_{2}, 0 \leq s_{1}+s_{2}, \\
1+\frac{1}{q}+\frac{s}{n}=\frac{1}{q_{1}}+\frac{s_{1}}{n}+\frac{1}{q_{2}}+\frac{s_{2}}{n}, \\
\frac{1}{q}+\frac{s}{n}<\frac{1}{q_{1}}+\frac{s_{1}}{n}, \frac{1}{q}+\frac{s}{n}<\frac{1}{q_{2}}+\frac{s_{2}}{n}, \frac{1}{q}+\frac{s}{n}>0 .
\end{array}\right.
$$

The set $X$ now is the union of mutually disjoint sets $X_{j}$ :

$$
X=\bigcup_{j=1}^{4} X_{j}
$$

where $X_{i} \cap X_{j}=\emptyset$ whenever $i \neq j$.

To prove Theorem 1.1, it suffices to show $A \subset X$ and $A \cap X_{j}=A_{j}$. Then the conclusion of Theorem 1.1 follows from the easy fact

$$
A=A \cap X=A \cap\left(\bigcup_{i=1}^{4} X_{i}\right)=\bigcup_{i=1}^{4}\left(A \cap X_{i}\right)=\bigcup_{i=1}^{4} A_{i} .
$$

\subsection{The proof of $A \subset X$.}

We define two sequences $\left\{a_{k, N}\right\}_{k \in \mathbb{Z}^{n}}$ and $\left\{b_{k, N}\right\}_{k \in \mathbb{Z}^{k}}$ for each natural number $N$, where

$$
a_{k, N}=\left\{\begin{array}{ll}
1, & \text { for }|k| \leq N, \\
0, & \text { otherwise },
\end{array} \quad b_{k, N}= \begin{cases}1, & \text { for }|k| \leq 2 N \\
0, & \text { otherwise }\end{cases}\right.
$$

For $k \in \mathbb{Z}^{n},|k| \leq N$, we have

$$
\sum_{j \in \mathbb{Z}^{n}} a_{k-j, N} b_{j, N} \geq \sum_{|j| \leq 2 N} a_{k-j, N} \geq \sum_{|j| \leq N} a_{j, N} \geq N^{n},
$$

and

$$
\left\|\left\{a_{k, N}\right\} *\left\{b_{k, N}\right\}\right\|_{l(q, s)} \geq N^{n}\left(\sum_{|k| \leq N}\langle k\rangle^{s q}\right)^{1 / q} .
$$


So, by the definition of $A$ we deduce

$$
N^{n}\left(\sum_{|k| \leq N}\langle k\rangle^{s q}\right)^{1 / q} \lesssim\left(\sum_{|k| \leq N}\langle k\rangle^{s_{1} q_{1}}\right)^{1 / q_{1}}\left(\sum_{|k| \leq 2 N}\langle k\rangle^{s_{2} q_{2}}\right)^{1 / q_{2}}
$$

For any $\delta>0$, we have

$$
N^{n[(1 / q+s / n) \vee 0]-\delta} \lesssim\left(\sum_{|k| \leq N}\langle k\rangle^{s q}\right)^{1 / q} \lesssim N^{n[(1 / q+s / n) \vee 0]+\delta}
$$

Combining it with (3.1), we then have that

$$
N^{n} \cdot N^{n[(1 / q+s / n) \vee 0]-\delta} \lesssim N^{n\left[\left(1 / q_{1}+s_{1} / n\right) \vee 0\right]+\delta} \cdot N^{n\left[\left(1 / q_{2}+s_{2} / n\right) \vee 0\right]+\delta}
$$

as $N \rightarrow \infty$, which implies

$$
n+n\left[\left(\frac{1}{q}+\frac{s}{n}\right) \vee 0\right]-\delta \leq n\left[\left(\frac{1}{q_{1}}+\frac{s_{1}}{n}\right) \vee 0\right]+\delta+n\left[\left(\frac{1}{q_{2}}+\frac{s_{2}}{n}\right) \vee 0\right]+\delta
$$

Letting $\delta \rightarrow 0$, we further obtain

$$
1+\left(\frac{1}{q}+\frac{s}{n}\right) \vee 0 \leq\left(\frac{1}{q_{1}}+\frac{s_{1}}{n}\right) \vee 0+\left(\frac{1}{q_{2}}+\frac{s_{2}}{n}\right) \vee 0 .
$$

On the other hand, we may take $b_{0}=1$ and $b_{k}=0(k \neq 0)$ in the inequality

$$
\left\|\left\{a_{k}\right\} *\left\{b_{k}\right\}\right\|_{l(q, s)} \lesssim\left\|\left\{a_{k}\right\}\right\|_{l\left(q_{1}, s_{1}\right)}\left\|\left\{b_{k}\right\}\right\|_{l\left(q_{2}, s_{2}\right)}
$$

to deduce

$$
\left\|\left\{a_{k}\right\}\right\|_{l(q, s)} \lesssim\left\|\left\{a_{k}\right\}\right\|_{l\left(q_{1}, s_{1}\right)}
$$

which implies that

$$
l\left(q_{1}, s_{1}\right) \subset l(q, s) .
$$

A similar argument then gives the inclusion

$$
l\left(q_{2}, s_{2}\right) \subset l(q, s) .
$$

Using an easy dual argument, we have

$$
\left\|\left\{a_{k}\right\} *\left\{b_{k}\right\}\right\|_{l\left(q_{1}^{\prime},-s_{1}\right)} \lesssim\left\|\left\{a_{k}\right\}\right\|_{l\left(q^{\prime},-s\right)}\left\|\left\{b_{k}\right\}\right\|_{l\left(q_{2}, s_{2}\right)} .
$$

In fact, by the assumption $l\left(q_{1}, s_{1}\right) * l\left(q_{2}, s_{2}\right) \subset l(q, s)$, we obtain

$$
\begin{aligned}
\left|\sum_{k \in \mathbb{Z}^{n}} \sum_{j \in \mathbb{Z}^{n}} c_{k-j} b_{j} a_{k}\right| & \leqslant\left\|\left\{c_{k}\right\} *\left\{b_{k}\right\}\right\|_{l(q, s)}\left\|\left\{a_{k}\right\}\right\|_{l\left(q^{\prime},-s\right)} \\
& \leqslant\left\|\left\{c_{k}\right\}\right\|_{l\left(q_{1}, s_{1}\right)}\left\|\left\{b_{k}\right\}\right\|_{l\left(q_{2}, s_{2}\right)}\left\|\left\{a_{k}\right\}\right\|_{l\left(q^{\prime},-s\right)},
\end{aligned}
$$


where we use the Hölder inequality in the first inequality. Observing that

$$
\sum_{k \in \mathbb{Z}^{n}} \sum_{j \in \mathbb{Z}^{n}} c_{k-j} b_{j} a_{k}=\sum_{k \in \mathbb{Z}^{n}} \sum_{j \in \mathbb{Z}^{n}} c_{j} b_{k-j} a_{k}=\sum_{j \in \mathbb{Z}^{n}} c_{j} \sum_{k \in \mathbb{Z}^{n}} b_{k-j} a_{k}
$$

we actually have

$$
\left|\sum_{j \in \mathbb{Z}^{n}} c_{j} \sum_{k \in \mathbb{Z}^{n}} b_{k-j} a_{k}\right| \leqslant\left\|\left\{c_{k}\right\}\right\|_{l\left(q_{1}, s_{1}\right)}\left\|\left\{b_{k}\right\}\right\|_{l\left(q_{2}, s_{2}\right)}\left\|\left\{a_{k}\right\}\right\|_{l\left(q^{\prime},-s\right)} .
$$

By the arbitrary of $\left\{c_{k}\right\}_{k \in \mathbb{Z}^{n}}$, we obtain

$$
\left\|\left\{\sum_{k \in \mathbb{Z}^{n}} b_{k-j} a_{k}\right\}\right\|_{l_{j}\left(q_{1}^{\prime},-s_{1}\right)} \leqslant\left\|\left\{b_{k}\right\}\right\|_{l\left(q_{2}, s_{2}\right)}\left\|\left\{a_{k}\right\}\right\|_{l\left(q^{\prime},-s\right)} .
$$

Rewriting $b_{j}=\tilde{b}_{-j}$, we obtain $\sum_{k \in \mathbb{Z}^{n}} b_{k-j} a_{k}=\left(\left\{a_{k}\right\} *\left\{b_{k}\right\}\right)(j)$. Then

$$
\begin{aligned}
\left\|\left\{a_{k}\right\} *\left\{\tilde{b}_{k}\right\}\right\|_{l\left(q_{1}^{\prime},-s_{1}\right)} & \leqslant\left\|\left\{b_{k}\right\}\right\|_{l\left(q_{2}, s_{2}\right)}\left\|\left\{a_{k}\right\}\right\|_{l\left(q^{\prime},-s\right)} \\
& =\left\|\left\{a_{k}\right\}\right\|_{l\left(q^{\prime},-s\right)}\left\|\left\{\tilde{b}_{k}\right\}\right\|_{l\left(q_{2}, s_{2}\right)},
\end{aligned}
$$

which is just the inequality (3.3).

Proceed the argument as above, we obtain that

$$
l\left(q_{2}, s_{2}\right) \subset l\left(q_{1}^{\prime},-s_{1}\right) .
$$

Also, invoking Proposition 2.3, we can get

$$
\frac{1}{q}+\frac{s}{n} \leq \frac{1}{q_{1}}+\frac{s_{1}}{n}, \frac{1}{q}+\frac{s}{n} \leq \frac{1}{q_{2}}+\frac{s_{2}}{n}, \frac{1}{q_{1}^{\prime}}+\frac{-s_{1}}{n} \leq \frac{1}{q_{2}}+\frac{s_{2}}{n},
$$

and

$$
s \leq s_{1}, \quad s \leq s_{2}, \quad-s_{1} \leq s_{2},
$$

where

$$
\left\{\begin{array}{l}
(q, s)=\left(q_{1}, s_{1}\right), \text { if } \frac{1}{q}+\frac{s}{n}=\frac{1}{q_{1}}+\frac{s_{1}}{n} \\
(q, s)=\left(q_{2}, s_{2}\right), \text { if } \frac{1}{q}+\frac{s}{n}=\frac{1}{q_{2}}+\frac{s_{2}}{n} \\
\left(q_{1}^{\prime},-s_{1}\right)=\left(q_{2}, s_{2}\right), \text { if } \frac{1}{q_{1}^{\prime}}+\frac{-s_{1}}{n}=\frac{1}{q_{2}}+\frac{s_{2}}{n} .
\end{array}\right.
$$

We emphasize that, in our proofs in this paper, many endpoint cases will be reduced to an embedding relations satisfying the condition such as the if parts in (3.4), then by using Proposition 2.3, the two function spaces in the corresponding embedding relations are actually the same. We have now completed the proof of $A \subset X$. 


\subsection{The proof of $A \cap X_{1}=A_{1}$.}

In order to show $A \cap X_{1}=A_{1}$, we need the following proposition for reduction purpose.

Proposition 3.1 (For reduction, discrete form). Suppose $0<q, q_{1}, q_{2} \leq \infty$, $s>0$. If

$$
\frac{1}{q} \leq \frac{1}{q_{1}}, \frac{1}{q} \leq \frac{1}{q_{2}}, \quad 1+\frac{1}{q}<\frac{1}{q_{1}}+\frac{1}{q_{2}}+\frac{s}{n}
$$

then

$$
l\left(q_{1}, s\right) * l\left(q_{2}, s\right) \subset l(q, s) .
$$

Proof. By the assumption, we have

$$
\frac{1}{q_{1}}+\frac{1}{q_{2}}-\frac{1}{q} \geq 0
$$

We denote

$$
\frac{1}{r}=\left(\frac{1}{q_{1}}+\frac{1}{q_{2}}-\frac{1}{q}\right) \wedge 1
$$

and let $t=r^{\prime}$. Then $r \in[1, \infty]$ and

$$
\frac{1}{r}+\frac{1}{q} \leq \frac{1}{q_{1}}+\frac{1}{q_{2}}
$$

Using Proposition 2.7, we deduce that

$$
\left\|\frac{\langle k\rangle^{s}}{\langle k-j\rangle^{s}\langle j\rangle^{s}}\right\|_{l_{j}^{t}} \lesssim 1
$$

uniformly for $k \in \mathbb{Z}^{n}$.

For $r \neq \infty$,

$$
\begin{aligned}
& \left(\sum_{k \in \mathbb{Z}^{n}}\left(\sum_{j \in \mathbb{Z}^{n}} a_{k-j} b_{j}\right)^{q}\langle k\rangle^{s q}\right)^{1 / q} \\
= & \left(\sum_{k \in \mathbb{Z}^{n}}\left(\sum_{j \in \mathbb{Z}^{n}} a_{k-j}\langle k-j\rangle^{s} b_{j}\langle j\rangle^{s} \frac{\langle k\rangle^{s}}{\langle k-j\rangle^{s}\langle j\rangle^{s}}\right)^{q}\right)^{1 / q} \\
\lesssim & \sup _{k \in \mathbb{Z}^{n}}\left\|\frac{\langle k\rangle^{s}}{\langle k-j\rangle^{s}\langle j\rangle^{s}}\right\|_{l_{j}^{t}}\left(\sum_{k \in \mathbb{Z}^{n}}\left(\sum_{j \in \mathbb{Z}^{n}} a_{k-j}^{r}\langle k-j\rangle^{s r} b_{j}^{r}\langle j\rangle^{s r}\right)^{q / r}\right)^{1 / q} \\
\lesssim & \left(\sum_{k \in \mathbb{Z}^{n}}\left(\sum_{j \in \mathbb{Z}^{n}} a_{k-j}^{r}\langle k-j\rangle^{s r} b_{j}^{r}\langle j\rangle^{s r}\right)^{q / r}\right)^{1 / q}=\left\|\left\{a_{k}^{r}\langle k\rangle^{s r}\right\} *\left\{b_{k}^{r}\langle k\rangle^{s r}\right\}\right\|_{l^{q / r}}^{1 / r} .
\end{aligned}
$$


By the fact that $1 / q \leq 1 / q_{1}, 1 / q \leq 1 / q_{2}$ and $1 / r+1 / q \leq 1 / q_{1}+1 / q_{2}$, we have, by Proposition 2.5 ,

$$
\begin{aligned}
\left\|\left\{a_{k}^{r}\langle k\rangle^{s r}\right\} *\left\{b_{k}^{r}\langle k\rangle^{s r}\right\}\right\|_{l^{q / r}}^{1 / r} & \lesssim\left\|\left\{a_{k}^{r}\langle k\rangle^{s r}\right\}\right\|_{l^{q_{1} / r}}^{1 / r}\left\|\left\{b_{k}^{r}\langle k\rangle^{s r}\right\}\right\|_{l^{q_{2} / r}}^{1 / r} \\
& =\left\|\left\{a_{k}\langle k\rangle^{s}\right\}\right\|_{l^{q_{1}}}\left\|\left\{b_{k}\langle k\rangle^{s}\right\}\right\|_{l^{q_{2}}} \\
& =\left\|\left\{a_{k}\right\}\right\|_{l\left(q_{1}, s\right)}\left\|\left\{b_{k}\right\}\right\|_{l\left(q_{2}, s\right)} .
\end{aligned}
$$

For $r=\infty$, we have $q=q_{1}=q_{2}=\infty$ and

$$
\left\|\frac{\langle k\rangle^{s}}{\langle k-j\rangle^{s}\langle j\rangle^{s}}\right\|_{l_{j}^{1}} \lesssim 1
$$

uniformly for $k \in \mathbb{Z}^{n}$. So for any $k \in \mathbb{Z}^{n}$, we have

$$
\begin{aligned}
\sum_{j \in \mathbb{Z}^{n}} a_{k-j} b_{j}\langle k\rangle^{s} & =\sum_{j \in \mathbb{Z}^{n}} a_{k-j}\langle k-j\rangle^{s} b_{j}\langle j\rangle^{s} \frac{\langle k\rangle^{s}}{\langle k-j\rangle^{s}\langle j\rangle^{s}} \\
& \lesssim\left\|\left\{a_{k}\right\}\right\|_{l(\infty, s)}\left\|\left\{b_{k}\right\}\right\|_{l(\infty, s)} \sum_{j \in \mathbb{Z}^{n}} \frac{\langle k\rangle^{s}}{\langle k-j\rangle^{s}\langle j\rangle^{s}} \\
& \lesssim\left\|\left\{a_{k}\right\}\right\|_{l(\infty, s)}\left\|\left\{b_{k}\right\}\right\|_{l(\infty, s)} .
\end{aligned}
$$

Hence

$$
\left\|\left\{a_{k}\right\} *\left\{b_{k}\right\}\right\|_{l(\infty, s)} \lesssim\left\|\left\{a_{k}\right\}\right\|_{l(\infty, s)}\left\|\left\{b_{k}\right\}\right\|_{l(\infty, s)}
$$

which completes the proof of Proposition 3.1.

Now we can return to prove $A \cap X_{1}=A_{1}$. First, the inclusion $A \cap X_{1} \subset A_{1}$ is obvious, since this fact can be verified directly by

$$
A \cap X_{1}=A \cap A_{1} \subset A_{1}
$$

It remains to show $A_{1} \subset A \cap X_{1}$. To this end, we only need to show

$$
(\mathbf{q}, \mathbf{s}) \in A_{1} \Longrightarrow l\left(q_{1}, s_{1}\right) * l\left(q_{2}, s_{2}\right) \subset l(q, s)
$$

We will consider three different cases:

Case $1: s \geq 0, s_{1} \geq 0, s_{2} \geq 0$;

Case 2: $s<0, s_{1}>0, s_{2}>0$;

Case 3: $s<0, s_{1} \leq 0, s_{2} \geq 0$ or $s<0, s_{1} \geq 0, s_{2} \leq 0$.

We point out that Case 2 and Case 3 can be reduced to Case 1 .

In fact, in Case 2 one can choose

$$
\frac{1}{\bar{q}}=\max \left\{\frac{1}{q}+\frac{s}{n}, 0\right\}, \bar{s}>0,
$$


so that, by Proposition 2.3 ,

$$
l(\bar{q}, \bar{s}) \subset l(q, s)
$$

and the new index group $(\overline{\mathbf{q}}, \overline{\mathbf{s}})=\left(\bar{q}, q_{1}, q_{2}, \bar{s}, s_{1}, s_{2}\right)$ belongs to Case 1 . Hence, the conclusion with Case 2 can be deduced by that with Case 1 and the embedding of $l(\bar{q}, \bar{s})$ and $l(q, s)$.

In Case 3 we may assume $s<0, s_{1} \leq 0, s_{2} \geq 0$ for the symmetry of $s_{1}, s_{2}$. We now easily verify that

$$
\left(\frac{1}{q_{1}}+\frac{s_{1}}{n}\right) \vee 0+\left(\frac{1}{q_{1}^{\prime}}+\frac{-s_{1}}{n}\right) \vee 0 \leq\left(\frac{1}{q}+\frac{s}{n}\right) \vee 0+\left(\frac{1}{q^{\prime}}+\frac{-s}{n}\right) \vee 0 .
$$

Combining it with

$$
1+\left(\frac{1}{q}+\frac{s}{n}\right) \vee 0<\left(\frac{1}{q_{1}}+\frac{s_{1}}{n}\right) \vee 0+\left(\frac{1}{q_{2}}+\frac{s_{2}}{n}\right) \vee 0
$$

we have

$$
1+\left(\frac{1}{q_{1}^{\prime}}+\frac{-s_{1}}{n}\right) \vee 0<\left(\frac{1}{q^{\prime}}+\frac{-s}{n}\right) \vee 0+\left(\frac{1}{q_{2}}+\frac{s_{2}}{n}\right) \vee 0 .
$$

The new index $(\overline{\overline{\mathbf{q}}}, \overline{\overline{\mathbf{s}}})=\left(q_{1}^{\prime}, q^{\prime}, q_{2},-s_{1},-s, s_{2}\right)$ then belongs to Case 1 . If one can deduce

$$
l\left(q^{\prime},-s\right) * l\left(q_{2}, s_{2}\right) \subset l\left(q_{1}^{\prime},-s_{1}\right)
$$

from Case 1, the conclusion associated with Case 3 follows easily by a dual argument.

Now, we only need to handle Case 1. By the spirit of Proposition 2.3, one can choose $\widetilde{q_{1}}, \widetilde{q_{2}} \in(0, \infty]$, such that

$$
\frac{1}{\widetilde{q_{1}}}+\frac{s}{n} \leqslant \frac{1}{q_{1}}+\frac{s_{1}}{n}
$$

with strict inequality if $s<s_{1}$,

$$
\frac{1}{\widetilde{q_{2}}}+\frac{s}{n} \leqslant \frac{1}{q_{2}}+\frac{s_{2}}{n}
$$

with strict inequality if $s<s_{2}$, and

$$
\left\{\begin{array}{l}
s \geq 0, \\
\frac{1}{q}+\frac{s}{n} \leq \frac{1}{\widetilde{q_{1}}}+\frac{s}{n}, \frac{1}{q}+\frac{s}{n} \leq \frac{1}{\widetilde{q_{2}}}+\frac{s}{n}, \\
1+\frac{1}{q}+\frac{s}{n}<\frac{1}{\widetilde{q_{1}}}+\frac{s}{n}+\frac{1}{\widetilde{q_{2}}}+\frac{s}{n} .
\end{array}\right.
$$

Then, by Proposition 2.3, we obtain

$$
l\left(q_{1}, s_{1}\right) \subset l\left(\widetilde{q_{1}}, s\right), l\left(q_{2}, s_{2}\right) \subset l\left(\widetilde{q_{2}}, s\right) .
$$

Moreover, we can use Proposition 2.5 or Proposition 3.1 to deduce

$$
l\left(\widetilde{q_{1}}, s\right) * l\left(\widetilde{q_{2}}, s\right) \subset l(q, s) .
$$

The desired conclusion now follows by an embedding argument. 


\subsection{The proof of $A \cap X_{2}=A_{2}$.}

We want to show the inclusion $A \cap X_{2} \subset A_{2}$.

Firstly, we show

$(\mathbf{q}, \mathbf{s}) \in A \cap X_{2} \cap\left\{(\mathbf{q}, \mathbf{s}) \in X_{2}: \frac{1}{q}+\frac{s}{n}=\frac{1}{q_{1}}+\frac{s_{1}}{n}\right\} \Longrightarrow\left\{\begin{array}{l}s=s_{1}=s_{2}=0, \\ q=q_{1}, q_{2}=1 .\end{array}\right.$

In this case, we have $(q, s)=\left(q_{1}, s_{1}\right)$ and

$$
\frac{1}{q_{2}}+\frac{s_{2}}{n}=1 \text {. }
$$

Using (1.8) and Hölder's inequality, we obtain

$$
\begin{aligned}
\sum_{k \in \mathbb{Z}^{n}} \sum_{j \in \mathbb{Z}^{n}} a_{j} b_{k-j} c_{k} & \lesssim\left\|\left\{a_{k}\right\} *\left\{b_{k}\right\}\right\|_{l(q, s)}\left\|\left\{c_{k}\right\}\right\|_{l\left(q^{\prime},-s\right)} \\
& \lesssim\left\|\left\{a_{k}\right\}\right\|_{l\left(q_{1}, s_{1}\right)}\left\|\left\{c_{k}\right\}\right\|_{l\left(q^{\prime},-s\right)}\left\|\left\{b_{k}\right\}\right\|_{l\left(q_{2}, s_{2}\right)} .
\end{aligned}
$$

For $\frac{1}{q_{1}}+\frac{s_{1}}{n}>0$, we let

$$
a_{k, N}=\left\{\begin{array}{ll}
\frac{1}{(2 N)^{s_{1}+n / q_{1}}}, & |k| \leq 2 N \\
0, & \text { otherwise, }
\end{array} \quad c_{k, N}= \begin{cases}\frac{\langle k\rangle^{t}}{N^{t-s+n / q^{r}}}, & |k| \leq 2 N \\
0, & \text { otherwise }\end{cases}\right.
$$

for some $t$ satisfying

$$
t>-n, \frac{1}{q^{\prime}}+\frac{t-s}{n}>0 .
$$

It is easy to see that

$$
\left\|\left\{a_{k, N}\right\}\right\|_{l\left(q_{1}, s_{1}\right)} \sim\left\|c_{k, N}\right\|_{l\left(q^{\prime},-s\right)} \sim 1 .
$$

We then use (3.5) to deduce

$$
\begin{aligned}
\left\|\left\{b_{k}\right\}\right\|_{l\left(q_{2}, s_{2}\right)} & \gtrsim \sum_{k \in \mathbb{Z}^{n}} \sum_{j \in \mathbb{Z}^{n}} a_{j, N} b_{k-j} c_{k, N} \\
& \gtrsim \frac{1}{(2 N)^{s_{1}+\frac{n}{q_{1}}}} \sum_{|k| \leq N} c_{k, N} \sum_{|j| \leq 2 N} b_{k-j} \\
& \gtrsim \frac{1}{(2 N)^{s_{1}+\frac{n}{q_{1}}}} \sum_{|k| \leq N} c_{k, N} \sum_{|j| \leq N} b_{j} \\
& \sim \frac{1}{(2 N)^{s_{1}+\frac{n}{q_{1}}}} \cdot \frac{N^{t+n}}{N^{t-s+\frac{n}{q^{\prime}}}} \sum_{|j| \leq N} b_{j} \sim \sum_{|j| \leq N} b_{j} .
\end{aligned}
$$

Letting $N \rightarrow \infty$, we obtain the embedding relationship

$$
l\left(q_{2}, s_{2}\right) \subset l(1,0),
$$


and use Proposition 2.3 to deduce

$$
s_{2}=0, q_{2}=1
$$

Recalling $0 \leq s_{1}+s_{2}, s=s_{1}, s \leq s_{2}$, we deduce

$$
s=s_{1}=s_{2}=0
$$

For $\frac{1}{q_{1}}+\frac{s_{1}}{n} \leq 0$, we have $\frac{1}{q^{\prime}}+\frac{-s}{n} \geq 1>0$. We can rewrite

$$
\sum_{j \in \mathbb{Z}^{n}} a_{j} \sum_{k \in \mathbb{Z}^{n}} c_{k} b_{k-j}=\sum_{k \in \mathbb{Z}^{n}} c_{k} \sum_{j \in \mathbb{Z}^{n}} a_{j} b_{k-j}
$$

and use the same argument as above to deduce the conclusion.

Next, we can show that

$(\mathbf{q}, \mathbf{s}) \in A \cap X_{2} \cap\left\{(\mathbf{q}, \mathbf{s}) \in X_{2}: \frac{1}{q}+\frac{s}{n}=\frac{1}{q_{2}}+\frac{s_{2}}{n}\right\} \Longrightarrow\left\{\begin{array}{l}s=s_{1}=s_{2}=0 \\ q=q_{2}, q_{1}=1\end{array}\right.$

by using the same argument above.

Finally, we show

$(\mathbf{q}, \mathbf{s}) \in A \cap X_{2} \cap\left\{(\mathbf{q}, \mathbf{s}) \in X_{2}: \frac{1}{q}+\frac{s}{n}=0\right\} \Longrightarrow\left\{\begin{array}{l}s=s_{1}=s_{2}=0 \\ q=\infty, \frac{1}{q_{1}}+\frac{1}{q_{2}}=1 .\end{array}\right.$

In this case, we have

$$
1=\frac{1}{q_{1}}+\frac{s_{1}}{n}+\frac{1}{q_{2}}+\frac{s_{2}}{n} \Longleftrightarrow \frac{1}{q_{1}^{\prime}}+\frac{-s_{1}}{n}=\frac{1}{q_{2}}+\frac{s_{2}}{n} .
$$

Also, we have

$$
\frac{1}{q^{\prime}}+\frac{-s}{n}=1
$$

So, a dual argument gives that

$$
l\left(q^{\prime},-s\right) * l\left(q_{2}, s_{2}\right) \subset l\left(q_{1}^{\prime},-s_{1}\right) .
$$

With the same argument as we used above, we obtain the facts

$$
-s_{1}=-s=-s_{2}=0, q^{\prime}=1, q_{2}=q_{1}^{\prime}
$$

We have now completed the proof for $A_{2} \supset A \cap X_{2}$.

To prove the opposite inclusion $A_{2} \subset A \cap X_{2}$, we need to show

$$
(\mathbf{q}, \mathbf{s}) \in A_{2} \Longrightarrow l\left(q_{1}, s_{1}\right) * l\left(q_{2}, s_{2}\right) \subset l(q, s) .
$$

This fact can be directly verified by Proposition 2.5. 


\subsection{The proof of $A \cap X_{3}=A_{3}$.}

We want to prove the inclusion $A \cap X_{3} \subset A_{3}$. We only need to show

$$
(\mathbf{q}, \mathbf{s}) \in A \cap X_{3} \Longrightarrow\left\{\begin{array}{l}
\frac{1}{q_{1}}+\frac{1}{q_{2}}=1, s_{1}+s_{2}=0 \\
0 \leq \frac{1}{q_{1}}+\frac{s_{1}}{n}, \frac{1}{q_{2}}+\frac{s_{2}}{n} .
\end{array}\right.
$$

In this case, we have

$$
1=\left(\frac{1}{q_{1}}+\frac{s_{1}}{n}\right) \vee 0+\left(\frac{1}{q_{2}}+\frac{s_{2}}{n}\right) \vee 0 .
$$

Hence, the inequalities

$$
1 \leq \frac{1}{q_{1}}+\frac{s_{1}}{n}+\frac{1}{q_{2}}+\frac{s_{2}}{n} \leq\left(\frac{1}{q_{1}}+\frac{s_{1}}{n}\right) \vee 0+\left(\frac{1}{q_{2}}+\frac{s_{2}}{n}\right) \vee 0=1
$$

implies

$$
1=\frac{1}{q_{1}}+\frac{s_{1}}{n}+\frac{1}{q_{2}}+\frac{s_{2}}{n}=\left(\frac{1}{q_{1}}+\frac{s_{1}}{n}\right) \vee 0+\left(\frac{1}{q_{2}}+\frac{s_{2}}{n}\right) \vee 0 .
$$

So the desired conclusion holds.

To show the opposite inclusion $A_{3} \subset A \cap X_{3}$, it suffices to show

$$
(\mathbf{q}, \mathbf{s}) \in A_{3} \Longrightarrow l\left(q_{1}, s_{1}\right) * l\left(q_{2}, s_{2}\right) \subset l(q, s)
$$

In fact, we have

$$
1+\left(\frac{1}{q_{1}^{\prime}}+\frac{-s_{1}}{n}\right) \vee 0<\left(\frac{1}{q^{\prime}}+\frac{-s}{n}\right) \vee 0+\left(\frac{1}{q_{2}}+\frac{s_{2}}{n}\right) \vee 0
$$

in this case. Hence the proof of (3.6) can be completed by a dual argument and the same method we used in the proof of $A \cap X_{1}=A_{1}$.

\subsection{The proof of $A \cap X_{4}=A_{4}$.}

We want to prove the inclusion $A \cap X_{4} \subset A_{4}$.

Firstly, we show

$$
(\mathbf{q}, \mathbf{s}) \in A \cap X_{4} \Longrightarrow \frac{1}{q} \leq \frac{1}{q_{1}}+\frac{1}{q_{2}} .
$$

The proof for case $q=\infty$ is obvious, so we only treat the case $q<\infty$. 
Fix $N \in \mathbb{N}$. Let

$$
a_{k, N}=\left\{\begin{array}{ll}
\langle k\rangle^{-s_{1}-\frac{n}{q_{1}}}, & |k| \leq N, \\
0, & \text { otherwise, }
\end{array} \quad b_{k, N}= \begin{cases}\langle k\rangle^{-s_{2}-\frac{n}{q_{2}}}, & |k| \leq N \\
0, & \text { otherwise }\end{cases}\right.
$$

It is easy to see that

$$
\left\|\left\{a_{k, N}\right\}\right\|_{l\left(q_{1}, s_{1}\right)} \sim(\ln N)^{\frac{1}{q_{1}}},\left\|\left\{b_{k, N}\right\}\right\|_{l\left(q_{2}, s_{2}\right)} \sim(\ln N)^{\frac{1}{q_{2}}} .
$$

On the other hand,

$$
\begin{aligned}
\left\|\left\{a_{k, N}\right\} *\left\{b_{k, N}\right\}\right\|_{l(q, s)}^{q} & =\sum_{k \in \mathbb{Z}^{n}}\left(\sum_{j \in \mathbb{Z}^{n}} a_{k-j, N} b_{j, N}\right)^{q}\langle k\rangle^{s q} \\
& =\sum_{|k| \leq N}\left(\sum_{|j| \leq N} a_{k-j, N} b_{j, N}\right)^{q}\langle k\rangle^{s q} \\
& \gtrsim \sum_{|k| \leq N}\left(\sum_{\left|j-\frac{1}{2} k\right| \leq\left|\frac{1}{4} k\right|}\langle k-j\rangle^{-s_{1}-\frac{n}{q_{1}}}\langle j\rangle^{-s_{2}-\frac{n}{q_{2}}}\right)^{q}\langle k\rangle^{s q} \\
& \gtrsim \sum_{|k| \leq N}\left(\sum_{\left|j-\frac{1}{2} k\right| \leq\left|\frac{1}{4} k\right|}\langle k\rangle^{-s_{1}-\frac{n}{q_{1}}-s_{2}-\frac{n}{q_{2}}}\right)^{q}\langle k\rangle^{s q} \\
& \gtrsim \sum_{|k| \leq N}\left(\langle k\rangle^{n} \cdot\langle k\rangle^{-s_{1}-\frac{n}{q_{1}}-s_{2}-\frac{n}{q_{2}}}\right)^{q}\langle k\rangle^{s q} .
\end{aligned}
$$

Recalling

$$
1+\frac{1}{q}+\frac{s}{n}=\frac{1}{q_{1}}+\frac{s_{1}}{n}+\frac{1}{q_{2}}+\frac{s_{2}}{n}
$$

we deduce that

$$
\left\|\left\{a_{k, N}\right\} *\left\{b_{k, N}\right\}\right\|_{l(q, s)}^{q} \gtrsim \sum_{|k| \leq N}\left(\langle k\rangle^{-s-n / q}\right)^{q}\langle k\rangle^{s q}=\sum_{|k| \leq N}\langle k\rangle^{-n} \sim \ln N
$$

which gives

$$
\left\|\left\{a_{k, N}\right\} *\left\{b_{k, N}\right\}\right\|_{l(q, s)} \gtrsim(\ln N)^{1 / q} .
$$

Hence, the obtained inequality

$$
(\ln N)^{1 / q} \lesssim(\ln N)^{1 / q_{1}} \cdot(\ln N)^{1 / q_{2}}
$$

implies that

$$
\frac{1}{q} \leq \frac{1}{q_{1}}+\frac{1}{q_{2}}
$$

Now, we show

$(\mathbf{q}, \mathbf{s}) \in A \cap X_{4} \cap\left\{(\mathbf{q}, \mathbf{s}) \in X_{4}: s=s_{1}\right.$ or $\left.s=s_{2}\right\} \Longrightarrow q \neq \infty, q_{1}, q_{2} \neq 1$. 
We will prove this fact by a contradiction argument. If $(\mathbf{q}, \mathbf{s})$ satisfies

$$
\left\{\begin{array}{l}
0<s \leq s_{1}, s=s_{2} \\
1=\frac{1}{q_{1}}+\frac{1}{q_{2}}+\frac{s_{1}}{n}, q=\infty \\
\frac{s}{n}<\frac{1}{q_{1}}+\frac{s_{1}}{n}, q_{2}<\infty
\end{array}\right.
$$

We define

$$
a_{k}=\langle k\rangle^{-n / q_{1}-s_{1}}(1+\ln \langle k\rangle)^{\epsilon_{1}}
$$

for all $k \in \mathbb{Z}^{n}$, where

$$
\epsilon_{1}= \begin{cases}\text { a real number such that } q_{1} \epsilon_{1}<-1, & \text { if } q_{1}<\infty \\ 0, & \text { if } q_{1}=\infty\end{cases}
$$

For any $N \in \mathbb{Z}^{n}$, we define

$$
b_{k, N}=\frac{\langle N-k\rangle^{-\frac{n}{q_{2}}}}{\langle k\rangle^{s_{2}}}(1+\ln \langle N-k\rangle)^{\epsilon_{2}}
$$

for all $k \in \mathbb{Z}^{n}$, where $\epsilon_{2}$ is a real number to be chosen later such that $q_{2} \epsilon_{2}<-1$. By a direct calculation, we have $\left\{a_{k}\right\}_{k \in \mathbb{Z}^{n}} \in l\left(q_{1}, s_{1}\right)$, and

$$
\left\|\left\{b_{k, N}\right\}\right\|_{l\left(q_{2}, s_{2}\right)} \lesssim 1
$$

uniformly for all $N \in \mathbb{Z}^{n}$. But we find

$$
\begin{aligned}
& \sum_{|j-N| \leq|N| / 2} a_{N-j} b_{j, N}\langle N\rangle^{s} \\
= & \sum_{|j-N| \leq|N| / 2}\langle N-j\rangle^{-n / q_{1}-s_{1}}(1+\ln \langle N-j\rangle)^{\epsilon_{1}+\epsilon_{2}} \cdot \frac{\langle N-j\rangle^{-n / q_{2}}}{\langle j\rangle^{s_{2}}}\langle N\rangle^{s} \\
\sim & \sum_{|j-N| \leq|N| / 2}\langle N-j\rangle^{-n / q_{1}-s_{1}}(1+\ln \langle N-j\rangle)^{\epsilon_{1}+\epsilon_{2}} \cdot\langle N-j\rangle^{-n / q_{2}} \\
= & \sum_{|j| \leq|N| / 2}\langle j\rangle^{-n / q_{1}-s_{1}}(1+\ln \langle j\rangle)^{\epsilon_{1}+\epsilon_{2}} \cdot\langle j\rangle^{-n / q_{2}} \\
= & \sum_{|j| \leq|N| / 2}\langle j\rangle^{-n}(1+\ln \langle j\rangle)^{\epsilon_{1}+\epsilon_{2}} .
\end{aligned}
$$

Since (3.7) implies

$$
\frac{1}{q_{1}}+\frac{1}{q_{2}}<1
$$

we can choose $\epsilon_{1}$ and $\epsilon_{2}$ such that

$$
\epsilon_{1}+\epsilon_{2}>-1 \text {. }
$$


On the other hand, it is easy to check that

$$
\left\|\left\{a_{k}\right\}\right\|_{l\left(q_{1}, s_{1}\right)} \cdot\left\|\left\{b_{k, N}\right\}\right\|_{l\left(q_{2}, s_{2}\right)} \lesssim 1
$$

uniformly on $N$. This leads to a contradiction

$$
\begin{aligned}
1 \gtrsim\left\|\left\{a_{k}\right\} *\left\{b_{k, N}\right\}\right\|_{l(\infty, s)} & \geq\left(\left\{a_{k}\right\} *\left\{b_{k, N}\right\}\right)(N)\langle N\rangle^{s} \\
& =\sum_{j \in \mathbb{Z}^{n}} a_{N-j} b_{j, N}\langle N\rangle^{s} \\
& \gtrsim \sum_{|j-N| \leq \frac{|N|}{2}} a_{N-j} b_{j, N}\langle N\rangle^{s} \\
& \gtrsim \sum_{|j| \leq \frac{|N|}{2}}\langle j\rangle^{-n}(1+\ln \langle j\rangle)^{\epsilon_{1}+\epsilon_{2}} \rightarrow \infty
\end{aligned}
$$

as $|N| \rightarrow \infty$.

If $s=s_{1}, q=\infty$, one can also deduce a contradiction by the same argument as above. Also, the case $q_{1}=1$ or $q_{2}=1$ can be handled by a dual argument.

To complete the proof of $A_{4}=A \cap X_{4}$, it now remains to show $A_{4} \subset$ $A \cap X_{4}$.

Firstly, we show

$(\mathbf{q}, \mathbf{s}) \in A_{4} \cap\left\{(\mathbf{q}, \mathbf{s}) \in A_{4}: q \neq \infty, q_{1}, q_{2} \neq 1\right\} \Longrightarrow l\left(q_{1}, s_{1}\right) * l\left(q_{2}, s_{2}\right) \subset l(q, s)$.

This conclusion will be obtained by using the fact that the discrete form of the Young-type inequalities can be deduced from the continuous form of Young-type inequalities. To this end, we establish the following proposition which will also play a pivotal action in the proof of Theorem 1.2 .

Proposition 3.2 (Implication method). Suppose $1 \leq q, q_{1}, q_{2} \leq \infty, s, s_{1}, s_{2} \in$ $\mathbb{R}$. Then

$$
\mathbb{L}\left(q_{1}, s_{1}\right) * \mathbb{L}\left(q_{2}, s_{2}\right) \subset \mathbb{L}(q, s)
$$

implies

$$
l\left(q_{1}, s_{1}\right) * l\left(q_{2}, s_{2}\right) \subset l(q, s) .
$$

Proof. We denote the unit cube

$$
Q=\left\{x \in \mathbb{R}^{n}:-1 / 2 \leq x_{j} \leq 1 / 2, j=1,2, \cdots, n\right\} .
$$

Given two positive sequences $\left\{a_{k}\right\}_{k \in \mathbb{Z}^{n}}$ and $\left\{b_{k}\right\}_{k \in \mathbb{Z}^{n}}$ defined on $\mathbb{Z}^{n}$, we define the companion functions

$$
f(x)=\sum_{k \in \mathbb{Z}^{n}} a_{k} \chi_{Q \backslash \frac{1}{8} Q}(x-k)
$$


and

$$
g(x)=\sum_{k \in \mathbb{Z}^{n}} b_{k} \chi_{Q \backslash \frac{1}{8} Q}(x-k) .
$$

It is easy to check that

$$
\|f\|_{\mathbb{L}\left(q_{1}, s_{1}\right)} \sim\left(\sum_{k \in \mathbb{Z}^{n}} a_{k}^{q_{1}}\langle k\rangle^{s_{1} q_{1}}\right)^{1 / q_{1}}=\left\|\left\{a_{k}\right\}\right\|_{l_{k}\left(q_{1}, s_{1}\right)}
$$

and

$$
\|g\|_{\mathbb{L}\left(q_{2}, s_{2}\right)} \sim\left(\sum_{k \in \mathbb{Z}^{n}} b_{k}^{q_{2}}\langle k\rangle^{s_{2} q_{2}}\right)^{1 / q_{2}}=\left\|\left\{b_{k}\right\}\right\|_{l_{k}\left(q_{2}, s_{2}\right)} .
$$

On the other hand,

$$
\begin{aligned}
& \|f * g\|_{\mathbb{L}(q, s)}^{q}=\int_{\mathbb{R}^{n}}\left|\int_{\mathbb{R}^{n}} f(x-y) g(y) d y\right|^{q}|x|^{s q} d x \\
= & \sum_{k \in \mathbb{Z}^{n}} \int_{Q+k}\left|\sum_{j \in \mathbb{Z}^{n}} \int_{Q+j} f(x-y) g(y) d y\right|^{q}|x|^{s q} d x \\
= & \sum_{k \in \mathbb{Z}^{n}} \int_{Q}\left|\sum_{j \in \mathbb{Z}^{n}} \int_{Q} f(k-j+x-y) g(y+j) d y\right|^{q}|x+k|^{s q} d x \\
\gtrsim & \sum_{k \in \mathbb{Z}^{n}} \int_{\frac{1}{2} Q}\left|\sum_{j \in \mathbb{Z}^{n}} \int_{\frac{1}{2} Q} f(k-j+x-y) g(y+j) d y\right|^{q}|x+k|^{s q} d x \\
= & \sum_{k \in \mathbb{Z}^{n}} \int_{\frac{1}{2} Q}\left|\sum_{j \in \mathbb{Z}^{n}} b_{j} \int_{\frac{1}{2} Q \backslash \frac{1}{8} Q} f(k-j+x-y) d y\right|^{q}|x+k|^{s q} d x \\
\geq & \sum_{k \in \mathbb{Z}^{n}} \int_{\frac{1}{2} Q}\left|\sum_{j \in \mathbb{Z}^{n}} a_{k-j} b_{j} \int_{\left(\frac{1}{2} Q \backslash \frac{1}{8} Q\right) \cap\left(x+Q \backslash \frac{1}{8} Q\right)} d y\right|^{q}|x+k|^{s q} d x \\
= & \sum_{k \in \mathbb{Z}^{n}} \int_{\frac{1}{2} Q}\left|\sum_{j \in \mathbb{Z}^{n}} a_{k-j} b_{j}\right|\left(\frac{1}{2} Q \backslash \frac{1}{8} Q\right) \cap\left(x+Q \backslash \frac{1}{8} Q\right)||^{q}|x+k|^{s q} d x \\
\gtrsim & \sum_{k \in \mathbb{Z}^{n}} \int_{\frac{1}{2} Q}\left|\sum_{j \in \mathbb{Z}^{n}} a_{k-j} b_{j}\right|^{q}|x+k|^{s q} d x \\
\gtrsim & \sum_{k \in \mathbb{Z}^{n}}\left|\sum_{j \in \mathbb{Z}^{n}} a_{k-j} b_{j}\right|^{q}\langle k\rangle^{s q}=\left\|\left\{a_{k}\right\} *\left\{b_{k}\right\}\right\|_{l(q, s)}^{q} \cdot
\end{aligned}
$$

Now the desired conclusion $l\left(q_{1}, s_{1}\right) * l\left(q_{2}, s_{2}\right) \subset l(q, s)$ follows directly from the above proposition and Theorem A.

Next, we show

$$
(\mathbf{q}, \mathbf{s}) \in A_{4} \cap\left\{(\mathbf{q}, \mathbf{s}) \in A_{4}: q=\infty, s<s_{1}, s<s_{2}\right\}
$$


implies

$$
l\left(q_{1}, s_{1}\right) * l\left(q_{2}, s_{2}\right) \subset l(\infty, s) .
$$

In this case, we have

$$
s>0, \frac{1}{q_{1}}+\frac{1}{q_{2}}<1 .
$$

We denote

$$
\frac{1}{t}=1-\frac{1}{q_{1}}-\frac{1}{q_{2}}=\frac{s_{1}+s_{2}-s}{n} .
$$

For any $k \in \mathbb{Z}^{n}$, we use Proposition 2.7 to deduce

$$
\begin{aligned}
\sum_{j \in \mathbb{Z}^{n}} a_{k-j} b_{j}\langle k\rangle^{s} & =\sum_{j \in \mathbb{Z}^{n}} a_{k-j}\langle k-j\rangle^{s_{1}} b_{j}\langle j\rangle^{s_{2}} \frac{\langle k\rangle^{s}}{\langle k-j\rangle^{s_{1}}\langle j\rangle^{s_{2}}} \\
& \lesssim\left\|\left\{a_{k}\langle k\rangle^{s_{1}}\right\}\right\|_{l^{q_{1}}} \cdot\left\|\left\{b_{k}\langle k\rangle^{s_{2}}\right\}\right\|_{l^{q_{2}}} \cdot\left\|\left\{\frac{\langle k\rangle^{s}}{\langle k-j\rangle^{s_{1}}\langle j\rangle^{s_{2}}}\right\}\right\|_{l_{j}^{t}} \\
& \lesssim\left\|\left\{a_{k}\right\}\right\|_{l\left(q_{1}, s_{1}\right)} \cdot\left\|\left\{b_{k}\right\}\right\|_{l\left(q_{2}, s_{2}\right)} .
\end{aligned}
$$

Finally, by a dual argument, we can deduce the conclusion in the case $q_{1}=1$ or $q_{2}=1, s<s_{1}, s<s_{2}$. The proof of Theorem 1.1 is completed.

\section{Continuous weighted Young's inequality- Proof of Theorem 1.2 and Theorem 1.3.}

\subsection{The proof of Theorem 1.2 .}

We start our proof by showing the necessity

$$
\mathbb{L}\left(\mathbf{q}_{\mathbf{1}}, \mathbf{s}_{\mathbf{1}}\right) * \mathbb{L}\left(\mathbf{q}_{\mathbf{2}}, \mathbf{s}_{\mathbf{2}}\right) \subset \mathbb{L}(\mathbf{q}, \mathbf{s}) \Longrightarrow(\mathbf{q}, \mathbf{s}) \in A_{2} \cup A_{4} .
$$

Using a dilation argument, we first deduce

$$
1+\frac{1}{q}+\frac{s}{n}=\frac{1}{q_{1}}+\frac{s_{1}}{n}+\frac{1}{q_{2}}+\frac{s_{2}}{n} .
$$

Then, we choose

$$
f(x)=\chi_{B(-2,1)}, g(x)=\chi_{B(2,1)} .
$$

Observing

$$
(f * g)(x) \gtrsim 1
$$

for $x \in B(0,1 / 2)$, we obtain

$$
\begin{aligned}
\int_{B(0,1 / 2)}|x|^{q s} d x & \lesssim \int_{B(0,1 / 2)}(f * g)^{q}(x)|x|^{q s} d x \\
& \lesssim\|f * g\|_{\mathbb{L}(q, s)}^{q} \lesssim\|f\|_{\mathbb{L}\left(q_{1}, s_{1}\right)}^{q} \cdot\|g\|_{\mathbb{L}\left(q_{2}, s_{2}\right)}^{q} \lesssim 1
\end{aligned}
$$


for $q<\infty$. It clearly yields

$$
\begin{cases}\frac{1}{q}+\frac{s}{n}>0, & \text { if } q<\infty \\ s \geq 0, & \text { if } q=\infty\end{cases}
$$

On the other hand, by Proposition 3.2 and Theorem 1.1, we know

$$
(\mathbf{q}, \mathbf{s}) \in \bigcup_{i=1}^{4} A_{i}
$$

Combining with (4.1), (4.2) and (4.3), we conclude

$$
(\mathbf{q}, \mathbf{s}) \in A_{2} \cup A_{4} \text {. }
$$

To prove the sufficiency of Theorem 1.2

$$
(\mathbf{q}, \mathbf{s}) \in A_{2} \cup A_{4} \Longrightarrow \mathbb{L}\left(\mathbf{q}_{\mathbf{1}}, \mathbf{s}_{\mathbf{1}}\right) * \mathbb{L}\left(\mathbf{q}_{\mathbf{2}}, \mathbf{s}_{\mathbf{2}}\right) \subset \mathbb{L}(\mathbf{q}, \mathbf{s})
$$

we only need to handle the case $q=\infty$ or $q_{1}=1$ or $q_{2}=1$ in $\mathcal{A}_{4}$. The other cases can be deduced directly by the classical Young's inequality and Kerman's result (See Theorem A).

By a dual method, we only need to show the proof for $q=\infty$. In this case, we have

$$
s<s_{1}, s<s_{2}
$$

and

$$
1=\frac{1}{q_{1}}+\frac{1}{q_{2}}+\frac{s_{1}+s_{2}-s}{n} .
$$

We now use Proposition 2.9 to deduce

$$
\begin{aligned}
& \int_{\mathbb{R}^{n}} f(x-y) g(y)|x|^{s} d y \\
= & \int_{\mathbb{R}^{n}} f(x-y)|x-y|^{s_{1}} g(y)|y|^{s_{2}} \frac{|x|^{s}}{|x-y|^{s_{1}}|y|^{s_{2}}} d y \\
\lesssim & \left\|f(x)|x|^{s_{1}}\right\|_{L_{x}^{q_{1}}} \cdot\left\|g(x)|x|^{s_{2}}\right\|_{L_{x}^{q_{2}}} \cdot\left\|\frac{|x|^{s}}{|x-y|^{s_{1}}|y|^{s_{2}}}\right\|_{L_{y}^{n /\left(s_{1}+s_{2}-s\right)}} \\
\lesssim & \|f\|_{\mathbb{L}\left(q_{1}, s_{1}\right)} \cdot\|g\|_{\mathbb{L}\left(q_{2}, s_{2}\right)} .
\end{aligned}
$$

\subsection{The proof of Theorem 1.3 .}

Firstly, we introduce some notations. Denote, for $i=1,2,3,4$,

$$
\begin{aligned}
B & =\left\{(\mathbf{q}, \mathbf{s}) \in[1, \infty]^{3} \times \mathbb{R}^{3}: L\left(q_{1}, s_{1}\right) * L\left(q_{2}, s_{2}\right) \subset L(q, s)\right\}, \\
B_{i} & =A_{i} \cap\left\{(\mathbf{q}, \mathbf{s}) \in[1, \infty]^{3} \times \mathbb{R}^{3}: 1+\frac{1}{q} \geq \frac{1}{q_{1}}+\frac{1}{q_{2}}\right\} .
\end{aligned}
$$


Using the same strategy for the proof of Theorem 1.1, we only need to show

$$
B=\bigcup_{i=1}^{4} B_{i}
$$

The inclusion $B \subset \bigcup_{i=1}^{4} B_{i}$ is based on the following two propositions.

Proposition 4.1 (Relationship between discrete and continuous weighted Young's inequality). Let $1 \leq q, q_{i} \leq \infty, s_{i} \in \mathbb{R}$, for $i=1,2$. Then the inclusion

$$
L\left(q_{1}, s_{1}\right) * L\left(q_{2}, s_{2}\right) \subset L(q, s)
$$

implies

$$
l\left(q_{1}, s_{1}\right) * l\left(q_{2}, s_{2}\right) \subset l(q, s) .
$$

Proof. One can verify this conclusion by the implication method, which we have used in Proposition 3.2. However, we here give another proof based on the pointview of the modulation spaces. In the definition of $V_{\phi} f$, since the choice of the window function is flexible, we choose two window functions $\phi_{1}$ and $\phi_{2}$ so that $\phi=\phi_{1} \phi_{2}$ is also a window function. An easy computation gives that

$$
V_{\phi}(f g)(x, \xi)=\left(V_{\phi_{1}} f(x, \cdot) * V_{\phi_{2}} g(x, \cdot)\right)(\xi) .
$$

By the Minkowski inequality and Hölder's inequality, we obtain

$$
\begin{aligned}
\|f g\|_{M_{1, q}^{s}} & =\left\|V_{\phi}(f g)(x, \xi)\right\|_{L_{\xi}(q, s)\left(L_{x}^{1}\right)} \\
& =\left\|\left(V_{\phi_{1}} f(x, \cdot) * V_{\phi_{2}} g(x, \cdot)\right)(\xi)\right\|_{L_{\xi}(q, s)\left(L_{x}^{1}\right)} \\
& \leq\left\|\int_{\mathbb{R}^{n}}\right\| V_{\phi_{1}} f(x, \xi-\eta) V_{\phi_{2}} g(x, \eta)\left\|_{L_{x}^{1}} d \eta\right\|_{L_{\xi}(q, s)} \\
& \leq\left\|\int_{\mathbb{R}^{n}}\right\| V_{\phi_{1}} f(x, \xi-\eta)\left\|_{L_{x}^{2}}\right\| V_{\phi_{2}} g(x, \eta)\left\|_{L_{x}^{2}} d \eta\right\|_{L_{\xi}(q, s)} .
\end{aligned}
$$

We now use the continuous weighted Young's inequality to deduce

$$
\begin{aligned}
\| \int_{\mathbb{R}^{n}} & \left\|V_{\phi_{1}} f(x, \xi-\eta)\right\|_{L_{x}^{2}}\left\|V_{\phi_{2}} g(x, \eta)\right\|_{L_{x}^{2}} d \eta \|_{L_{\xi}(q, s)} \\
& \lesssim\|\| V_{\phi_{1}} f(x, \xi)\left\|_{L_{x}^{2}}\right\|_{L_{\xi}\left(q_{1}, s_{1}\right)} \cdot\|\| V_{\phi_{2}} g(x, \xi)\left\|_{L_{x}^{2}}\right\|_{L_{\xi}\left(q_{2}, s_{2}\right)} \\
\quad & =\|f\|_{M_{2, q_{1}}^{s_{1}}} \cdot\|g\|_{M_{2, q_{2}}^{s_{2}}} \cdot
\end{aligned}
$$

In the next section, we will show Proposition 5.1, which says that the bound-

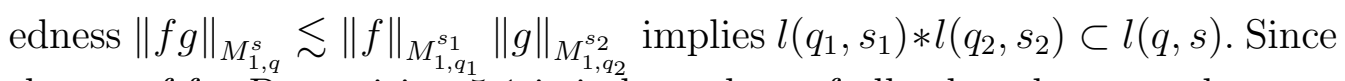
the proof for Proposition 5.1 is independent of all other theorems, here we bring it in advance to obtain Proposition 4.1. 
Proposition 4.2. Let $1 \leq q, q_{i} \leq \infty, s_{i} \in \mathbb{R}$, for $i=1,2$. Then

$$
L\left(q_{1}, s_{1}\right) * L\left(q_{2}, s_{2}\right) \subset L(q, s)
$$

implies

$$
1+\frac{1}{q} \geq \frac{1}{q_{1}}+\frac{1}{q_{2}}
$$

Proof. For $0<a<1$, we define

$$
f(x)=g(x)=\chi_{B(0, a)} .
$$

It is easy to verify that

$$
(f * g)(x) \gtrsim a^{n}
$$

for $x \in B\left(0, \frac{a}{2}\right)$. Also, a direct calculation shows that

$$
\|f\|_{L\left(q_{1}, s_{1}\right)} \sim a^{n / q_{1}},\|g\|_{L\left(q_{2}, s_{2}\right)} \sim a^{n / q_{2}}
$$

and

$$
\|f * g\|_{L(q, s)} \gtrsim a^{n+n / q} .
$$

as $a \rightarrow 0$. Hence, the inequality

$$
\|f * g\|_{L(q, s)} \lesssim\|f\|_{L\left(q_{1}, s_{1}\right)} \cdot\|g\|_{L\left(q_{2}, s_{2}\right)}
$$

implies

$$
a^{n+n / q} \lesssim a^{n / q_{1}} \cdot a^{n / q_{2}}
$$

The conclusion of the proposition now trivially follows by letting $a \rightarrow 0$ in the above inequality.

By Proposition 4.1, Proposition 4.2 and Theorem 1.1, we now obtain the inclusion

$$
B \subset \bigcup_{i=1}^{4} B_{i}
$$

Next, we want to show the opposite inclusion

$$
\bigcup_{i=1}^{4} B_{i} \subset B
$$

To this end, we need to show

$$
(\mathbf{q}, \mathbf{s}) \in \bigcup_{i=1}^{4} B_{i} \Longrightarrow L\left(q_{1}, s_{1}\right) * L\left(q_{2}, s_{2}\right) \subset L(q, s) .
$$


First, we show

$$
(\mathbf{q}, \mathbf{s}) \in B_{1} \Longrightarrow L\left(q_{1}, s_{1}\right) * L\left(q_{2}, s_{2}\right) \subset L(q, s) .
$$

Similar to the proof of Theorem 1.1, we divide the proof into three cases.

Case $1: s \geq 0, s_{1} \geq 0, s_{2} \geq 0$;

Case 2: $s<0, s_{1}>0, s_{2}>0$;

Case 3: $s<0, s_{1} \leq 0, s_{2} \geq 0$ or $s<0, s_{1} \geq 0, s_{2} \leq 0$.

Case 2 and Case 3 actually can be reduced to Case 1 with the following arguments.

In Case 2, we choose

$$
\frac{1}{\bar{q}}=\max \left\{\frac{1}{q}+\frac{s}{n}, \frac{1}{q_{1}}+\frac{1}{q_{2}}-1,0\right\} .
$$

Then

$$
\frac{1}{q} \geq \frac{1}{\bar{q}}, 1+\frac{1}{\bar{q}} \geq \frac{1}{q_{1}}+\frac{1}{q_{2}} .
$$

We can further choose $\bar{s}>0$ such that $1 / q+s / n<1 / \bar{q}+\bar{s} / n$, and then by Proposition 2.4, we have

$$
L(\bar{q}, \bar{s}) \subset L(q, s)
$$

and the new index $(\overline{\mathbf{q}}, \overline{\mathbf{s}})=\left(\bar{q}, q_{1}, q_{2}, \bar{s}, s_{1}, s_{2}\right)$ belongs to Case 1 . So the conclusion in Case 2 follows from Case 1 and (4.5).

On the other hand, Case 3 can be reduced to Case 1 by a dual argument, with a similar argument used in the proof of Theorem 1.1.

Before we handle Case 1, we also need the following proposition which is just a minor modification of Proposition 3.1. So we omit its proof.

Proposition 4.3 (For reduction, continuous form). Suppose $0<q, q_{1}, q_{2} \leq$ $\infty, s>0$. If

$$
\left\{\begin{array}{l}
s \leq s_{1}, \quad s \leq s_{2} \\
\frac{1}{q} \leq \frac{1}{q_{1}}, \frac{1}{q} \leq \frac{1}{q_{2}} \\
1+\frac{1}{q}+\frac{s}{n}<\frac{1}{q_{1}}+\frac{s_{1}}{n}+\frac{1}{q_{2}}+\frac{s_{2}}{n} \\
1+\frac{1}{q} \geq \frac{1}{q_{1}}+\frac{1}{q_{2}}
\end{array}\right.
$$

then we have

$$
L\left(q_{1}, s_{1}\right) * L\left(q_{2}, s_{2}\right) \subset L(q, s)
$$

Now we proceed the proof in Case 1. 
If $s>0$, we set

$$
\widetilde{q}_{i}= \begin{cases}q_{i}, & \text { if } \frac{1}{q} \leq \frac{1}{q_{i}} \\ q, & \text { if } \frac{1}{q}>\frac{1}{q_{i}}\end{cases}
$$

for $i=1,2$. Then we choose $\widetilde{s}_{i}$ such that $\tilde{s}_{i}=s_{i}$ for $1 / q \leqslant 1 / q_{i}$,

$$
1 / \tilde{q}_{i}+\tilde{s}_{i} / n<1 / q_{i}+s_{i} / n
$$

when $1 / q>1 / q_{i}$, and

$$
\left\{\begin{array}{l}
s \geq 0, s \leq \widetilde{s_{1}}, \quad s \leq \widetilde{s_{2}}, \\
\frac{1}{q} \leq \frac{1}{\widetilde{q_{1}}}, \frac{1}{q} \leq \frac{1}{\widetilde{q_{2}}} \\
1+\frac{1}{q}+\frac{s}{n}<\frac{1}{\widetilde{q_{1}}}+\frac{\widetilde{s_{1}}}{n}+\frac{1}{\widetilde{q_{2}}}+\frac{\widetilde{s_{2}}}{n} \\
1+\frac{1}{q} \geq \frac{1}{\widetilde{q_{1}}}+\frac{1}{\widetilde{q_{2}}} .
\end{array}\right.
$$

Using Proposition 2.4, we obtain following embedding relations:

$$
L\left(q_{1}, s_{1}\right) \subset L\left(\widetilde{q_{1}}, \widetilde{s_{1}}\right), L\left(q_{2}, s_{2}\right) \subset L\left(\widetilde{q_{2}}, \widetilde{s_{2}}\right)
$$

The conclusion (4.4) follows from the embedding relations (4.6) and Proposition 4.3.

If $s=0, s_{1}, s_{2}>0$, on can choose a small positive constant $\tilde{s}$, such that the new index group $\left(q, q_{1}, q_{2}, \tilde{s}, s_{1}, s_{2}\right)$ belongs to the previous case $s>0$. Thus, we have $L\left(q_{1}, s_{1}\right) * L\left(q_{2}, s_{2}\right) \subset L(q, \tilde{s}) \subset L(q, s)$.

If $s=s_{1}=0, s_{2}>0$, we have

$$
\left\{\begin{array}{l}
s=s_{1}=0, s_{2}>0 \\
1+\frac{1}{q}<\frac{1}{q_{1}}+\frac{1}{q_{2}}+\frac{s_{2}}{n} \\
\frac{1}{q} \leq \frac{1}{q_{1}}, \frac{1}{q}<\frac{1}{q_{2}}+\frac{s_{2}}{n} \\
1+\frac{1}{q} \geq \frac{1}{q_{1}}+\frac{1}{q_{2}}
\end{array}\right.
$$

Choose

$$
\frac{1}{r}=1+\frac{1}{q}-\frac{1}{q_{1}},
$$

and observe that

$$
\frac{1}{q_{2}} \leq \frac{1}{r} \leq 1, \frac{1}{r}<\frac{1}{q_{2}}+\frac{s_{2}}{n}
$$

Clearly, $r \in[1, \infty]$. We then use Young's inequality and the embedding relation $L\left(q_{2}, s_{2}\right) \subset L^{r}$ to deduce that

$$
\begin{aligned}
\|f * g\|_{L^{q}} & \lesssim\|f\|_{L^{q_{1}}} \cdot\|g\|_{L^{r}} \\
& \lesssim\|f\|_{L^{q_{1}}} \cdot\|g\|_{L\left(q_{2}, s_{2}\right)} .
\end{aligned}
$$

The case $s=s_{2}=0, s_{1}>0$ can be handled similarly. We have now completed the proof for (4.4). 
The proof of

$$
(\mathbf{q}, \mathbf{s}) \in B_{2} \Longrightarrow L\left(q_{1}, s_{1}\right) * L\left(q_{2}, s_{2}\right) \subset L(q, s)
$$

is a trivial one, we omit the details.

The proof for

$$
(\mathbf{q}, \mathbf{s}) \in B_{3} \Longrightarrow L\left(q_{1}, s_{1}\right) * L\left(q_{2}, s_{2}\right) \subset L(q, s)
$$

can proceed following the same method used in the proof of $A \cap X_{3}=A_{3}$, we also omit the details.

Finally, we show

$$
(\mathbf{q}, \mathbf{s}) \in B_{4} \Longrightarrow L\left(q_{1}, s_{1}\right) * L\left(q_{2}, s_{2}\right) \subset L(q, s) .
$$

If $q=\infty$ or $q_{1}=1$ or $q_{2}=1$, we can get the conclusion by the same method used in the proof of $A \cap X_{4}=A_{4}$ and Proposition 2.8. We only give the proof for $q=\infty$, since the other cases can be handled by a dual argument. If $q=\infty$, we have $s>0, s<s_{1}, s<s_{2}$. Take

$$
\frac{1}{t}=1-\frac{1}{q_{1}}-\frac{1}{q_{2}}=\frac{s_{1}+s_{2}-s}{n} .
$$

Using Proposition 2.8, we deduce that

$$
\begin{aligned}
& \left|\int_{\mathbb{R}^{n}} f(x-y) g(y) d y\langle x\rangle^{s}\right| \\
& \quad=\left|\int_{\mathbb{R}^{n}} f(x-y)\langle x-y\rangle^{s_{1}} g(y)\langle y\rangle^{s_{2}} \frac{\langle x\rangle^{s}}{\langle x-y\rangle^{s_{1}}\langle y\rangle^{s_{2}}} d y\right| \\
& \quad \lesssim\|f\|_{l\left(q_{1}, s_{1}\right)}\|g\|_{L\left(q_{2}, s_{2}\right)}\left\|\frac{\langle x\rangle^{s}}{\langle x-y\rangle^{s_{1}}\langle y\rangle^{s_{2}}}\right\|_{L_{y}^{t}} \\
& \quad \lesssim\|f\|_{l\left(q_{1}, s_{1}\right)}\|g\|_{L\left(q_{2}, s_{2}\right)} .
\end{aligned}
$$

It implies that $\|f * g\|_{L(\infty, s)} \lesssim\|f\|_{l\left(q_{1}, s_{1}\right)}\|g\|_{L\left(q_{2}, s_{2}\right)}$.

Next, we consider the case for $q \neq \infty, q_{1} \neq 1, q_{2} \neq 1$. By the symmetry of $s, s_{1}, s_{2}$, it suffices to handle the case $s_{1}, s_{2} \geq 0$. In this case, we have

$$
|x|^{s_{1}} \leq\langle x\rangle^{s_{1}},|x|^{s_{2}} \leq\langle x\rangle^{s_{2}},
$$

and

$$
\|f\|_{\mathbb{L}\left(q_{1}, s_{1}\right)} \leq\|f\|_{L\left(q_{1}, s_{1}\right)},\|g\|_{\mathbb{L}\left(q_{2}, s_{2}\right)} \leq\|g\|_{L\left(q_{2}, s_{2}\right)} .
$$

On the other hand, we write

$$
\begin{aligned}
\|f * g\|_{L(q, s)} \leq & \left(\int_{B(0,1)}|(f * g)(x)|^{q}\langle x\rangle^{s q} d x\right)^{1 / q} \\
& +\left(\int_{(B(0,1))^{c}}|(f * g)(x)|^{q}\langle x\rangle^{s q} d x\right)^{1 / q} \\
= & : \widetilde{I}+\widetilde{I I} .
\end{aligned}
$$


Using Theorem A, we have

$$
\begin{aligned}
\widetilde{I I} \sim\left(\int_{B^{c}(0,1)}|(f * g)(x)|^{q}|x|^{s q} d x\right)^{1 / q} & \lesssim\|f * g\|_{\mathbb{L}(q, s)} \\
& \lesssim\|f\|_{\mathbb{L}\left(q_{1}, s_{1}\right)} \cdot\|g\|_{\mathbb{L}\left(q_{2}, s_{2}\right)} \\
& \lesssim\|f\|_{L\left(q_{1}, s_{1}\right)} \cdot\|g\|_{L\left(q_{2}, s_{2}\right)} .
\end{aligned}
$$

For $\widetilde{I}$, if

$$
1+\frac{1}{q}>\frac{1}{q_{1}}+\frac{1}{q_{2}}
$$

one can choose

$$
0<\frac{1}{\widetilde{q}}<\frac{1}{q}
$$

so that

$$
\widetilde{I} \lesssim\left(\int_{B(0,1)}|(f * g)(x)|^{\widetilde{q}}\langle x\rangle^{\widetilde{s q}} d x\right)^{1 / \widetilde{q}} \leq\|f * g\|_{L(\widetilde{q}, s)}
$$

and the new index $(\widetilde{\mathbf{q}}, \widetilde{\mathbf{s}})=\left(\widetilde{q}, q_{1}, q_{2}, s, s_{1}, s_{2}\right)$ belongs to $B_{1}$. By the fact that $(\mathbf{q}, \mathbf{s}) \in B_{1} \Longrightarrow L\left(q_{1}, s_{1}\right) * L\left(q_{2}, s_{2}\right) \subset L(q, s)$, we deduce the inequality

$$
\|f * g\|_{L(\widetilde{q}, s)} \lesssim\|f\|_{L\left(q_{1}, s_{1}\right)} \cdot\|g\|_{L\left(q_{2}, s_{2}\right)} .
$$

Combining $\widetilde{I}$ with $\widetilde{I I}$, we obtain

$$
\|f * g\|_{L(q, s)} \lesssim \widetilde{I}+\widetilde{I I} \lesssim\|f\|_{L\left(q_{1}, s_{1}\right)} \cdot\|g\|_{L\left(q_{2}, s_{2}\right)}
$$

If

$$
1+\frac{1}{q}=\frac{1}{q_{1}}+\frac{1}{q_{2}}
$$

we have $s=s_{1}+s_{2}$, then $s=s_{1}=s_{2}=0$. It is a trivial case.

We complete the proof of Theorem 1.3 .

\section{Application on modulation spaces - Proof of Theorem 1.4 and 1.5}

\subsection{The proof of Theorem 1.4 .}

We first show the relationship between the product on modulation spaces and the discrete weighted Young's inequality. We need to establish the following proposition. 
Proposition 5.1 (Relationship between the product on modulation spaces and the discrete weighted Young's inequality). Let $1 \leq p, q, p_{i}, q_{i} \leq \infty, s_{i} \in$ $\mathbb{R}$, for $i=1,2$. Then

$$
\|f g\|_{M_{p, q}^{s}} \lesssim\|f\|_{M_{p_{1}, q_{1}}^{s_{1}}}\|g\|_{M_{p_{2}, q_{2}}^{s_{2}}}
$$

holds for $f, g \in \mathscr{S}\left(\mathbb{R}^{n}\right)$ if and only if

$$
\frac{1}{p} \leq \frac{1}{p_{1}}+\frac{1}{p_{2}}
$$

and

$$
l\left(q_{1}, s_{1}\right) * l\left(q_{2}, s_{2}\right) \subset l(q, s) .
$$

Proof. We first show the necessity part. Recalling that $\left\{\sigma_{k}\right\}_{k}$ is a smooth decomposition of $\mathbb{R}^{n}$ as defined in Section 2. We can choose a function $\hat{h}(\xi)$ in $C_{c}^{\infty}\left(\mathbb{R}^{n}\right)$ such that $\hat{h} \subset B(0,1 / 8)$, and that $\|h\|_{L^{p_{1}}},\|h\|_{L^{p_{2}}}$ and $\|h * h\|_{L^{p}}$ are not equal to 0 . Define

$$
\hat{v}_{\lambda}=\hat{h}(\xi / \lambda), 0 \leqslant \lambda \leqslant 1
$$

Observing that $\square_{0} v_{\lambda}=v_{\lambda}$ and $\square_{k} v_{\lambda}=v_{\lambda}$ for $k \neq 0$. Then

$$
\left\|v_{\lambda}\right\|_{M_{p, q}^{s}}=\left(\sum_{k \in \mathbb{Z}^{n}}\left\|\square_{k} v_{\lambda}\right\|_{L^{p}}^{q}\right)^{1 / q}=\left\|\square_{0} v_{\lambda}\right\|_{L^{p}}=\left\|v_{\lambda}\right\|_{L^{p}}
$$

Similarly, we obtain

$$
\left\|v_{\lambda} \cdot v_{\lambda}\right\|_{M_{p, q}^{s}}=\left\|v_{\lambda} \cdot v_{\lambda}\right\|_{L^{p}}
$$

Then we use the assumption

$$
\left\|v_{\lambda} \cdot v_{\lambda}\right\|_{M_{p, q}^{s}} \lesssim\left\|v_{\lambda}\right\|_{M_{p_{1}, q_{1}}^{s_{1}}}\left\|v_{\lambda}\right\|_{M_{p_{2}, q_{2}}^{s_{2}}}
$$

to deduce

$$
\begin{aligned}
\lambda^{n(2-1 / p)}\left\|h^{2}\right\|_{L^{p}} & \sim\left\|v_{\lambda} \cdot v_{\lambda}\right\|_{L^{p}} \\
& \lesssim\left\|v_{\lambda}\right\|_{L^{p_{1}}}\left\|v_{\lambda}\right\|_{L^{p_{2}}} \sim \lambda^{n\left(2-1 / p_{1}-1 / p_{2}\right)}
\end{aligned}
$$

Letting $\lambda \rightarrow 0$, we obtain

$$
\frac{1}{p} \leq \frac{1}{p_{1}}+\frac{1}{p_{2}}
$$

Denote

$$
\hat{h_{k}}(\xi)=\hat{h}(\xi-k)
$$


Let $a_{k}, b_{k} \in \mathbb{R}^{+}$for $k \in \mathbb{Z}^{n}$. By assuming that the following two series converge, we define two functions

$$
f(x)=\sum_{k \in \mathbb{Z}^{n}} a_{k} h(x) e^{2 \pi i k \cdot x}, \quad g(x)=\sum_{k \in \mathbb{Z}^{n}} b_{k} h(x) e^{2 \pi i k \cdot x} .
$$

We have

$$
(f g)(x)=\sum_{j, l \in \mathbb{Z}^{n}} a_{j} b_{l} h^{2}(x) e^{2 \pi i(j+l) \cdot x}
$$

Observing that

$$
\begin{aligned}
\left\|\square_{k}(f g)\right\|_{L^{p}} & =\left\|\sum_{j+l=k} a_{j} b_{l} h^{2}(x) e^{2 \pi i(j+l) \cdot x}\right\|_{L^{p}(d x)} \simeq \sum_{j+l=k} a_{j} b_{l}, \\
\left\|\square_{k}(f)\right\|_{L^{p_{1}}} & \simeq a_{k}, \quad\left\|\square_{k}(g)\right\|_{L^{p_{2}}} \simeq b_{k} \text { for all } k \in \mathbb{Z}^{n},
\end{aligned}
$$

by the definition of modulation space, we use the assumption

$$
\|f g\|_{M_{p, q}^{s}} \lesssim\|f\|_{M_{p_{1}, q_{1}}^{s_{1}}}\|g\|_{M_{p_{2}, q_{2}}^{s_{2}}}
$$

to deduce

$$
\left\|\left\{a_{k}\right\} *\left\{b_{k}\right\}\right\|_{l(q, s)} \lesssim\left\|\left\{a_{k}\right\}\right\|_{l\left(q_{1}, s_{1}\right)}\left\|\left\{b_{k}\right\}\right\|_{l\left(q_{2}, s_{2}\right)} .
$$

We turn to show the sufficiency of the proposition. Using the almost orthogonality of the frequency projections $\sigma_{k}$, we have that for all $k \in \mathbb{Z}^{n}$,

$$
\square_{k}(f g)=\sum_{i, j \in \mathbb{Z}^{n}} \square_{k}\left(\square_{i} f \cdot \square_{j} g\right)=\sum_{|l| \leq c(n)} \sum_{i+j=k+l} \square_{k}\left(\square_{i} f \cdot \square_{j} g\right),
$$

where $c(n)$ is a constant depending only on $n$. By the fact that $\square_{k}$ is an $L^{p}$ multiplier, we use Hölder's inequality and Lemma 2.2 to deduce that

$$
\begin{aligned}
\left\|\square_{k}(f g)\right\|_{L^{p}} & \lesssim\left\|\square_{k}(f g)\right\|_{L^{r}} \\
& =\left\|\sum_{|l| \leq c(n)} \sum_{i+j=k+l} \square_{k}\left(\square_{i} f \cdot \square_{j} g\right)\right\|_{L^{r}} \\
& \leq \sum_{|l| \leq c(n)} \sum_{i+j=k+l}\left\|\square_{i} f\right\|_{L^{p_{1}}}\left\|\square_{j} g\right\|_{L^{p_{2}}},
\end{aligned}
$$

where $1 / r=1 / p_{1}+1 / p_{2}$. Then the discrete weighted Young's inequality implies that

$$
\begin{aligned}
\|f g\|_{M_{p, q}^{s}} & =\left\|\left\{\left\|\square_{k}(f g)\right\|_{L^{p}}\right\}\right\|_{l(q, s)} \\
& \lesssim\left\|\left\{\left\|\square_{i} f\right\|_{L^{p_{1}}}\right\} *\left\{\left\|\square_{j} g\right\|_{L^{p_{2}}}\right\}\right\|_{l(q, s)} \\
& \lesssim\left\|\left\{\left\|\square_{k} f\right\|_{L^{p_{1}}}\right\}\right\|_{l\left(q_{1}, s_{1}\right)} \cdot\left\|\left\{\left\|\square_{k} g\right\|_{L^{p_{2}}}\right\}\right\|_{l\left(q_{2}, s_{2}\right)} \\
& =\|f\|_{M_{p_{1}, q_{1}}^{s_{1}}\|g\|_{M_{p_{2}, q_{2}}^{s_{2}}}}
\end{aligned}
$$

Proposition 5.1 is proved.

Now Theorem 1.4 can be verified immediately by the above Proposition 5.1 together with Theorem 1.1 . 


\subsection{The proof of Theorem 1.5}

To prove the necessity part, we take the special bilinear Fourier multiplier $T(f, g)=f g$. Choose

$$
p=1, p_{1}=2, p_{2}=2 \text {. }
$$

By Hölder's inequality,

$$
\|f g\|_{L^{p}} \lesssim\|f\|_{L^{p_{1}}}\|g\|_{L^{p_{2}}}
$$

Hence, using the assumption of $(\mathbf{q}, \mathbf{s})$ we have

$$
\|T(f, g)\|_{M_{p, q}^{s}}=\|f g\|_{M_{p, q}^{s}} \lesssim\|f\|_{M_{p_{1}, q_{1}}^{s_{1}}}\|g\|_{M_{p_{2}, q_{2}}^{s_{2}}} .
$$

Then the conclusion follows by Theorem 1.4.

For the sufficiency part, we notice that

$$
\square_{k}(T(f, g))=\sum_{i, j \in \mathbb{Z}^{n}} \square_{k}\left(T\left(\square_{i} f, \square_{j} g\right)\right)=\sum_{|l| \leq c(n)} \sum_{i+j=k+l} \square_{k}\left(T\left(\square_{i} f, \square_{j} g\right)\right) .
$$

Recall the assumption that $T: L^{p_{1}} \times L^{p_{2}} \rightarrow L^{p}$, then

$$
\left\|T\left(\square_{i} f, \square_{j} g\right)\right\|_{L^{p}} \lesssim\left\|\square_{i} f\right\|_{L^{p_{1}}}\left\|\square_{j} g\right\|_{L^{p_{2}}}
$$

Using the fact that $\square_{k}$ is an $L^{p}$ multiplier, we deduce that

$$
\begin{aligned}
\left\|\square_{k}(T(f, g))\right\|_{L^{p}} & =\left\|\sum_{|l| \leq c(n)} \sum_{i+j=k+l} \square_{k}\left(T\left(\square_{i} f, \square_{j} g\right)\right)\right\|_{L^{p}} \\
& \leq \sum_{|l| \leq c(n)} \sum_{i+j=k+l}\left\|\square_{i} f\right\|_{L^{p_{1}}}\left\|\square_{j} g\right\|_{L^{p_{2}}}
\end{aligned}
$$

as in the proof of (5.1). The rest part of this proof is the same as the proof of $(\underline{5.2})$.

\section{Fractional integral operators: Proof of The- orem 1.6 and Theorem 1.7 .}

\subsection{Proof of Theorem 1.6}

Firstly, we introduce some notations for simplicity. Denote

$$
C=\left\{(q, p, s, t) \in[1, \infty]^{2} \times \mathbb{R}^{2}: \mathcal{I}_{\lambda}: l(p, t) \rightarrow l(q, s)\right\}
$$


Use $C_{i}$ to denote the set of all $(q, p, s, t) \in[1, \infty]^{2} \times \mathbb{R}^{2}$ satisfying condition $\mathcal{C}_{i}$ mentioned in Theorem 1.6 respectively, $i=1,3,4$. Let $C_{2}=\emptyset$. We use $Z$ to denote the set of all $(\mathbf{q}, \mathbf{s}) \in[1, \infty]^{3} \times \mathbb{R}^{3}$ satisfying

$$
\left\{\begin{array}{l}
s \leq t \\
\frac{\lambda}{n}+\left(\frac{1}{q}+\frac{s}{n}\right) \vee 0 \leq\left(\frac{1}{p}+\frac{t}{n}\right) \vee 0 \\
\frac{\lambda}{n}+\frac{1}{q}+\frac{s}{n} \leq 1 \\
\left(q^{\prime},-s\right)=(1, \lambda-n) \text { if } \frac{\lambda}{n}+\frac{1}{q}+\frac{s}{n}=1 \\
(p, t)=(1, \lambda-n) \text { if } \frac{\lambda}{n}=\frac{1}{p}+\frac{t}{n}
\end{array}\right.
$$

We use $Z_{1}$ to denote the set of all $(\mathbf{q}, \mathbf{s}) \in Z$ satisfying

$$
\frac{\lambda}{n}+\left(\frac{1}{q}+\frac{s}{n}\right) \vee 0<\left(\frac{1}{p}+\frac{t}{n}\right) \vee 0 .
$$

Then $Z_{1}=C_{1}$. We also use $Z_{i}(i=2,3,4)$ to denote the sets of all $(\mathbf{q}, \mathbf{s}) \in Z$ satisfying

$$
\frac{\lambda}{n}+\left(\frac{1}{q}+\frac{s}{n}\right) \vee 0=\left(\frac{1}{p}+\frac{t}{n}\right) \vee 0,
$$

and

$$
\left\{\begin{array}{l}
\frac{1}{q}+\frac{s}{n}=0, \text { if } i=2 \\
\frac{1}{q}+\frac{s}{n}<0, \text { if } i=3 \\
\frac{1}{q}+\frac{s}{n}>0, \text { if } i=4
\end{array}\right.
$$

To prove the theorem, we will use the same strategy as before. We first show $C \subset Z$, then verify $C \cap Z_{j}=C_{j}$ for $j=1,2,3,4$. So, the conclusion follows from the easy fact

$$
C=C \cap Z=C \cap\left(\bigcup_{i=1}^{4} Z_{i}\right)=\bigcup_{i=1}^{4}\left(C \cap Z_{i}\right)=\bigcup_{i=1}^{4} C_{i} .
$$

To prove $C \subset Z$, for each positive integer $N$, choosing

$$
f_{N}(k)= \begin{cases}1, & \text { for }|k| \leq 2 N \\ 0, & \text { otherwise }\end{cases}
$$

we then have

$$
\begin{aligned}
\sum_{j \in \mathbb{Z}^{n}, j \neq k} \frac{f_{N}(j)}{|k-j|^{n-\lambda}} & \geq \sum_{|j| \leq 2 N, j \neq k} \frac{f_{N}(j)}{|k-j|^{n-\lambda}} \\
& \geq \sum_{|j| \leq 2 N, j \neq k} \frac{1}{|k-j|^{n-\lambda}} \\
& \geq \sum_{|j| \leq N, j \neq 0} \frac{1}{|j|^{n-\lambda}} \sim N^{\lambda}
\end{aligned}
$$


for $|k| \leq N$. So we get

$$
\left\|\mathcal{I}_{\lambda}\left(f_{N}\right)\right\|_{l(q, s)} \gtrsim N^{\lambda}\left(\sum_{|k| \leq N}\langle k\rangle^{s q}\right)^{1 / q} .
$$

By the similar argument used in Subsection 3.2, we can use the inequalities

$$
N^{\lambda}\left(\sum_{|k| \leq N}\langle k\rangle^{s q}\right)^{1 / q} \lesssim\left\|f_{N}\right\|_{l(p, t)} \lesssim\left(\sum_{|k| \leq 2 N}\langle k\rangle^{t p}\right)^{1 / p}
$$

to deduce

$$
\frac{\lambda}{n}+\left(\frac{1}{q}+\frac{s}{n}\right) \vee 0 \leq\left(\frac{1}{p}+\frac{t}{n}\right) \vee 0 .
$$

On the other hand, for nonnegative $f$, we use

$$
\left(\mathcal{I}_{\lambda} f\right)(k)=\sum_{j \in \mathbb{Z}^{n}, j \neq k} \frac{f(j)}{|k-j|^{n-\lambda}} \gtrsim f(k-1),
$$

to deduce

$$
\|f\|_{l(q, s)} \lesssim\left\|\mathcal{I}_{\lambda}(f)\right\|_{l(q, s)} \lesssim\|f\|_{l(p, t)}
$$

which implies

$$
l(p, t) \subset l(q, s) .
$$

Finally, Hölder's inequality yields that

$$
\sum_{k \in \mathbb{Z}^{n}} \sum_{j \in \mathbb{Z}^{n}, j \neq k} \frac{a_{j}}{|k-j|^{n-\lambda}} c_{k} \lesssim\left\|\left\{a_{k}\right\}\right\|_{l(p, t)}\left\|\left\{c_{k}\right\}\right\|_{l\left(q^{\prime},-s\right)} .
$$

Now we take $a_{0}=1$ and $a_{k}=0(k \neq 0)$ to obtain

$$
\sum_{k \in \mathbb{Z}^{n}, k \neq 0} \frac{c_{k}}{|k|^{n-\lambda}} \lesssim\left\|\left\{c_{k}\right\}\right\|_{l\left(q^{\prime},-s\right)}
$$

which clearly implies

$$
l\left(q^{\prime},-s\right) \subset l(1, \lambda-n) .
$$

Then, we take $c_{0}=1$ and $c_{k}=0(k \neq 0)$ to obtain

$$
l(p, t) \subset l(1, \lambda-n)
$$

The other conditions of $Z$ then can be verified by the above three embedding relations and Proposition 2.3 .

Next, we turn to show $C \cap Z_{j}=C_{j}$ for $j=1,2,3,4$.

First, to prove $C \cap Z_{1}=C_{1}$, we only need to show that

$$
(q, p, s, t) \in C_{1} \Longrightarrow \mathcal{I}_{\lambda}: l(p, t) \rightarrow l(q, s)
$$


We divide this part of proof into two cases.

Case 1: $\lambda / n+1 / q+s / n<1$. Using a similar argument as used in Subsection 3.3 , we only need to consider the case $s \geq 0$. Thus we have

$$
\left\{\begin{array}{l}
0 \leq s \leq t \\
1+\frac{1}{q}+\frac{s}{n}<\frac{1}{p}+\frac{t}{n}+\left(1-\frac{\lambda}{n}\right) \\
\frac{1}{q}+\frac{s}{n}<1-\frac{\lambda}{n}, \frac{1}{q}+\frac{s}{n}<\frac{1}{p}+\frac{t}{n}, 1<\frac{1}{p}+\frac{t}{n}+\left(1-\frac{\lambda}{n}\right) .
\end{array}\right.
$$

We can choose $q_{1} \in[1, \infty]$ such that

$$
\frac{1}{q}+\frac{s}{n}<\frac{1}{q_{1}}+\frac{s}{n}<1-\frac{\lambda}{n},
$$

and

$$
\left\{\begin{array}{l}
0 \leq s \leq t \\
1+\frac{1}{q}+\frac{s}{n}<\frac{1}{p}+\frac{t}{n}+\frac{1}{q_{1}}+\frac{s}{n} \\
\frac{1}{q}+\frac{s}{n}<\frac{1}{q_{1}}+\frac{s}{n}, \frac{1}{q}+\frac{s}{n}<\frac{1}{p}+\frac{t}{n}, 1<\frac{1}{p}+\frac{t}{n}+\frac{1}{q_{1}}+\frac{s}{n} .
\end{array}\right.
$$

By the fact

$$
\left\{\langle k\rangle^{\lambda-n}\right\}_{k \in \mathbb{Z}^{n}} \in l\left(q_{1}, s\right),
$$

we use Theorem 1.1 to deduce

$$
\begin{aligned}
\left\|\mathcal{I}_{\lambda}(f)\right\|_{l(q, s)} & \lesssim\left\|\left\{\langle k\rangle^{\lambda-n}\right\} * f\right\|_{l(q, s)} \\
& \lesssim\left\|\left\{\langle k\rangle^{\lambda-n}\right\}\right\|_{l\left(q_{1}, s\right)}\|f\|_{l(p, t)} \lesssim\|f\|_{l(p, t)} .
\end{aligned}
$$

Case 2: $\frac{\lambda}{n}+\frac{1}{q}+\frac{s}{n}=1$. In this case, we have $q=\infty, s=n-\lambda$.

$$
\begin{aligned}
\left\|\mathcal{I}_{\lambda}(f)\right\|_{l(\infty, n-\lambda)} & =\sup _{k \in \mathbb{Z}^{n}}\left(\sum_{j \in \mathbb{Z}^{n}, j \neq k} \frac{f(j)}{|k-j|^{n-\lambda}}\langle k\rangle^{n-\lambda}\right) \\
& \lesssim \sup _{k \in \mathbb{Z}^{n}}\left(\sum_{j \in \mathbb{Z}^{n}} f(j)\langle j\rangle^{t} \frac{\langle k\rangle^{n-\lambda}}{\langle k-j\rangle^{n-\lambda}\langle j\rangle^{t}}\right) \\
& \lesssim \sup _{k \in \mathbb{Z}^{n}}\left\|\frac{\langle k\rangle^{n-\lambda}}{\langle k-j\rangle^{n-\lambda}\langle j\rangle^{t}}\right\|_{l_{j}^{p^{\prime}}}\|f\|_{l(p, t)} .
\end{aligned}
$$

Observing that

$$
0<n-\lambda=s \leq t
$$

and

$$
1=\frac{\lambda}{n}+\frac{1}{q}+\frac{s}{n}<\frac{1}{p}+\frac{t}{n} \Rightarrow p^{\prime}>\frac{n}{t},
$$

we use Proposition 2.7 to deduce

$$
\sup _{k \in \mathbb{Z}^{n}}\left\|\frac{\langle k\rangle^{n-\lambda}}{\langle k-j\rangle^{n-\lambda}\langle j\rangle^{t}}\right\|_{l_{j}^{p^{\prime}}} \lesssim 1 .
$$


The desired conclusion $C \cap Z_{1}=C_{1}$ then follows.

Next, we claim $C \cap Z_{2}=\emptyset$. In fact, we have a special embedding relationship in this case. Firstly, we have $1 / p+t / n=\lambda / n$, then $p=1, t=\lambda-n$. For positive integer $N$, define

$$
a_{k, N}= \begin{cases}1, & \text { for }|k| \leq 2 N \\ 0, & \text { otherwise }\end{cases}
$$

It is easy to check that

$$
\left\|\left\{a_{k, N}\right\}\right\|_{l(p, t)} \sim N^{n / p+t}=N^{\lambda}
$$

and

$$
\begin{aligned}
\sum_{k \in \mathbb{Z}^{n}} \sum_{j \in \mathbb{Z}^{n}, j \neq k} \frac{a_{j, N}}{|k-j|^{n-\lambda}} c_{k} & \gtrsim \sum_{|k| \leq N} c_{k} \sum_{|j| \leq 2 N, j \neq k} \frac{a_{j, N}}{|k-j|^{n-\lambda}} \\
& \gtrsim \sum_{|k| \leq N} c_{k} \sum_{|j| \leq N, j \neq 0} \frac{1}{|j|^{n-\lambda}} \\
& \gtrsim N^{\lambda} \sum_{|k| \leq N} c_{k} .
\end{aligned}
$$

Then we use the assumption

$$
\sum_{k \in \mathbb{Z}^{n}} \sum_{j \in \mathbb{Z}^{n}, j \neq k} \frac{a_{j, N}}{|k-j|^{n-\lambda}} c_{k} \lesssim\left\|\left\{a_{k, N}\right\}\right\|_{l(p, t)}\left\|\left\{c_{k}\right\}\right\|_{l\left(q^{\prime},-s\right)}
$$

to deduce that

$$
N^{\lambda} \sum_{|k| \leq N} c_{k} \lesssim N^{\lambda}\left\|\left\{c_{k}\right\}\right\|_{l\left(q^{\prime},-s\right)}
$$

as $N \rightarrow \infty$. It gives the embedding

$$
l\left(q^{\prime},-s\right) \subset l(1,0)
$$

Using the fact $1 / q^{\prime}+(-s) / n=1$ and Proposition 2.3, we obtain

$$
q=\infty, s=0
$$

which contradicts the fact

$$
t=\lambda-n, s \leq t
$$

To prove $C \cap Z_{3}=C_{3}$, we first easily see that

$$
C \cap Z_{3} \subset C_{3}
$$


by the fact that $\frac{\lambda}{n}=\frac{1}{p}+\frac{t}{n}$, then $p=1, t=\lambda-n$.

To verify $C_{3} \subset C \cap Z_{3}$, it is sufficient to show

$$
(q, p, s, t) \in C_{3} \Longrightarrow \mathcal{I}_{\lambda}: l(p, t) \rightarrow l(q, s)
$$

However, this conclusion can be reduced to the $\mathcal{C}_{1}$ condition case by a dual argument. In fact, $\left(p^{\prime}, q^{\prime},-t,-s\right)$ belongs to $\mathcal{C}_{1}$. It implies that

$$
\mathcal{I}_{\lambda}: l\left(q^{\prime},-s\right) \rightarrow l\left(p^{\prime},-t\right)
$$

The desired conclusion $\mathcal{I}_{\lambda}: l(p, t) \rightarrow l(q, s)$ follows by a dual argument.

Our last step is to show $C \cap Z_{4}=C_{4}$.

To prove

$$
C \cap Z_{4} \subset C_{4}
$$

we first verify

$$
(q, p, s, t) \subset C \cap Z_{4} \Longrightarrow \frac{\lambda}{n}+\frac{1}{q}+\frac{s}{n} \neq 1
$$

In fact, if $\lambda / n+1 / q+s / n=1$, then we have

$$
\frac{1}{p^{\prime}}+\frac{-t}{n}=0, \frac{1}{q^{\prime}}+\frac{-s}{n}=\frac{\lambda}{n}
$$

which can be reduced to the proof of $C \cap Z_{2}=\emptyset$ by a dual argument.

Second, we need to show

$$
(q, p, s, t) \subset C \cap Z_{4} \Longrightarrow \frac{1}{q} \leq \frac{1}{p} .
$$

We define

$$
f_{N}(k)= \begin{cases}\langle k\rangle^{-t-n / p}, & \text { for }|k| \leq N \\ 0, & \text { otherwise }\end{cases}
$$

It is easy to check that

$$
\left\|\left\{f_{N}(k)\right\}\right\|_{l(p, t)}=(\ln N)^{1 / p} .
$$


On the other hand

$$
\begin{aligned}
\left\|\mathcal{I}_{\lambda}\left(f_{N}\right)\right\|_{l(q, s)} & =\left(\sum_{k \in \mathbb{Z}^{n}}\left(\sum_{j \in \mathbb{Z}^{n}, j \neq k} \frac{f_{N}(j)}{|k-j|^{n-\lambda}}\right)^{q}\langle k\rangle^{s q}\right)^{1 / q} \\
& \gtrsim\left(\sum_{|k| \leq|N|}\left(\sum_{|j-k| \leq \frac{|k|}{2}, j \neq k} \frac{f_{N}(j)}{|k-j|^{n-\lambda}}\right)^{q}\langle k\rangle^{s q}\right)^{1 / q} \\
& \gtrsim\left(\sum_{k \leq|N|}\left(\sum_{|j-k| \leq \frac{|k|}{2}, j \neq k} \frac{\langle k\rangle^{-t-\frac{n}{p}}}{|k-j|^{n-\lambda}}\right)^{q}\langle k\rangle^{s q}\right)^{1 / q} \\
& \gtrsim\left(\sum_{k \leq|N|}\left(\langle k\rangle^{\lambda}\langle k\rangle^{-t-\frac{n}{p}}\right)^{q}\langle k\rangle^{s q}\right)^{1 / q} \sim(\ln N)^{1 / q} .
\end{aligned}
$$

Then, we deduce the inequality

$$
(\ln N)^{1 / q} \lesssim(\ln N)^{1 / p}
$$

which implies the desired conclusion $1 / q \leqslant 1 / p$.

Finally, we want show that

$$
(q, p, s, t) \in C \cap Z_{4} \cap\left\{(q, p, s, t) \in Z_{4}: s=t\right\}
$$

implies $q \neq \infty, p \neq 1$.

If $s=t, q=\infty$, we have

$$
\frac{\lambda}{n}=\frac{1}{p}
$$

For any $N \in \mathbb{Z}^{n}$, we define

$$
g_{N}(k)=\frac{\langle k\rangle^{-t}}{\langle N-k\rangle^{\lambda}}(1+\ln \langle N-k\rangle)^{\epsilon}
$$

for all $k \in \mathbb{Z}^{n}$, where $\epsilon$ is a real number that satisfies $p \epsilon<-1$ and $\epsilon \geq-1$.

Now

$$
\begin{aligned}
\left\|\mathcal{I}_{\lambda}\left(g_{N}\right)\right\|_{l(\infty, s)} & =\sup _{k \in \mathbb{Z}^{n}}\left(\sum_{j \in \mathbb{Z}^{n}, j \neq k} \frac{g_{N}(j)}{|k-j|^{n-\lambda}}\langle k\rangle^{s}\right) \\
& \gtrsim \sum_{|j-N| \leq \frac{|N|}{2}, j \neq k} \frac{g_{N}(j)}{|N-j|^{n-\lambda}}\langle N\rangle^{s} \\
& \sim \sum_{|j-N| \leq \frac{|N|}{2}, j \neq k} \frac{\langle j\rangle^{-t}}{\langle N-j\rangle^{\lambda}}(1+\ln \langle N-j\rangle)^{\epsilon} \frac{1}{|N-j|^{n-\lambda}}\langle N\rangle^{s} \\
& \sim \sum_{|j-N| \leq \frac{|N|}{2}, j \neq k} \frac{1}{\langle N-j\rangle^{n}}(1+\ln \langle N-j\rangle)^{\epsilon} \rightarrow \infty
\end{aligned}
$$


as $N \rightarrow \infty$. This contradicts the fact that

$$
\left\|g_{N}\right\|_{l(p, t)} \lesssim 1
$$

uniformly as $N \rightarrow \infty$.

The case $s=t, p=1$ can be handled by a dual argument.

For the proof of

$$
C_{4} \subset C \cap Z_{4}
$$

we only need to show

$$
(q, p, s, t) \in C_{4} \Longrightarrow \mathcal{I}_{\lambda}: l(p, t) \rightarrow l(q, s)
$$

Based on the complete result (see Theorem 5 in [25]), using an adaptation of the implication method to the setting of boundedness of fractional integrals, we get this conclusion. In the endpoint case $p=1$ or $q=\infty$, we can also use Proposition 2.7 to verify this conclusion, just like we handled the same case in the proof of Theorem 1.1 .

\subsection{Proof of Theorem 1.7}

Since the sufficiency has been obtained by Strichartz [25], we only show that

$$
I_{\lambda}: \mathbb{L}(p, t) \rightarrow \mathbb{L}(q, s) \Longrightarrow(q, p, s, t) \in C_{4}
$$

By a dilation argument, we obtain

$$
\frac{\lambda}{n}+\frac{1}{q}+\frac{s}{n}=\frac{1}{p}+\frac{t}{n} .
$$

Hence, the only thing that we need to clarify is:

$$
\frac{1}{q}+\frac{s}{n} \geq 0
$$

Set

$$
f(x)=\chi_{B(2,1)}(x) .
$$

Then we have

$$
\|f\|_{\mathbb{L}(p, t)} \lesssim 1
$$

On the other hand,

$$
\begin{aligned}
\left\|I_{\lambda}(f)\right\|_{\mathbb{L}(q, s)} & \gtrsim\left\|I_{\lambda}(f) \chi_{B(0,1)}\right\|_{\mathbb{L}(q, s)} \\
& \gtrsim\left\|\chi_{B(0,1)}\right\|_{\mathbb{L}(q, s)} .
\end{aligned}
$$


So we should have

$$
\left\|\chi_{B(0,1)}(x)\right\|_{\mathbb{L}(q, s)} \lesssim 1
$$

which implies

$$
\frac{1}{q}+\frac{s}{n} \geq 0
$$

According to Theorem 1.6, we can use an implication argument (as Proposition 3.2) to deduce

$$
(q, p, s, t) \in \bigcup_{i=1,2,4} C_{i}
$$

Combining (6.1) with (6.2), we obtain

$$
(q, p, s, t) \in C_{4}
$$

Now, we complete the proof of Theorem 1.7 .

\section{Acknowledgements}

The authors sincerely appreciate the anonymous referee for checking this paper very carefully and giving very detailed comments, which greatly improved this article. This work was supported by the National Natural Sciences Foundation of China (Nos. 11371295, 11471041, 11471288, 11671414 and 11601456).

\section{References}

[1] A. Bényi, K. Gröchenig, K.A. Okoudjou and L.G. Rogers, Unimodular Fourier multiplier for modulation spaces, J. Funct. Anal. 246 (2007), 366-384.

[2] A. Biswas, D. Swanson, Navier-Stokes equations and weighted convolution inequalities in groups, Comm. Partial Differential Equations 35(4) (2010), 559-589.

[3] H.Q. Bui, Weighted Young's inequality and convolution theorems on weighted Besov spaces, Math. Nachr. 170 (1994), 25-37.

[4] E. Cordero, F. Nicola, Sharpness of some properties of Wiener Amalgam and modulaion spaces, Bull. Austral. Math. Soc. 80 (2009), 105116. 
[5] P.L. De Nápoli, I. Drelichman, R.G. Durán, On weighted inequalities for fractional integrals of radial functions, Illinois J. Math. 55(2) (2011), 575-587.

[6] J. Duoandikoetxea, Fractional integrals on radial functions with applications to weighted inequalities, Ann. Mat. Pura Appl. 192(4) (2013), $553-568$.

[7] H.G. Feichtinger, Modulation spaces on locally compact Abelian group, Technical Report, University of Vienna, 1983, in: "Proc. Inter. Conf. on Wavelet and Applications", 99-140, New Delhi Allied Publishers, India, 2003.

[8] H.G. Feichtinger, G. Narimani, Fourier multipliers of classical modulation spaces, Appl. Comput. Harmon. Anal. 21 (2006), 349-359.

[9] L. Grafakos, Modern Fourier Analysis: Structure of topological groups, Integration theory, Group representations, Graduate Texts in Mathematics, 250, Springer, 2009.

[10] T. Iwabuchi, Navier-Stokes equations and nonlinear heat equations in modulation spaces with negative derivative indices, J. Differential Equations 248(8) (2010), 1972-2002.

[11] R.A. Kerman, Convolution theorems with weights, Trans. Amer. Math. Soc. 280(1) (1983), 207-219.

[12] R. Kerman, E. Sawyer, Convolution algebras with weighted rearrangement-invariant norm, Studia Math. 108(2) (1994), 103-126.

[13] M. Lacey, K. Moen, C. Pérez, R.H. Torres, Sharp weighted bounds for fractional integral operators, J. Funct. Anal. 259(5) (2010), 1073-1097.

[14] B. Muckenhoupt, R. Wheeden, Weighted norm inequalities for fractional integrals, Trans. Amer. Math. Soc. 192 (1974), 261-274.

[15] A. Nowak, K. Stempak, Potential operators associated with Hankel and Hankel-Dunkl transforms, J. Danalyse Mathématique, 2017, 131(1), 277-321.

[16] E. Nursultanov and S. Tikhonov, Convolution inequalities in Lorentz spaces, J. Fourier Anal. Appl. 17(3) (2011), 486-505. 
[17] E. Nursultanov and S. Tikhonov, Weighted norm inequalities for convolution and Riesz potential, Potential Anal. 42(2) (2015), 435-456.

[18] B.S. Rubin, One-dimensional representation, inversion, and certain properties of the Riesz potentials of radial functions, Mat. Zametik 34(4) (1983), 521-533.

[19] M. Ruzhansky, M. Sugimoto, B. Wang, Modulation spaces and nonlinear evolution equations, Progr. Math. 301 (2012), 267-283.

[20] E. Sawyer, A two weight weak type inequality for fractional integrals, Trans. Amer. Math. Soc. 281 (1984), 339-345.

[21] E. Sawyer, A characterization of a two-weight norm inequality for fractional and Poisson integrals, Trans. Amer. Math. Soc. 308(2) (1988), 533-545.

[22] E.M. Stein, S. Wainger, Discrete analogues in harmonic analysis II: Fractional integration, J. Anal. Math. 80(1) (2000), 335-355.

[23] E.M. Stein, G. Weiss, Fractional integrals on n-dimensional Euclidean space, J. Math. Mech. 7 (1958), 503-514.

[24] E.M. Stein, Singular Integrals and Differentiability Properties of Functions, Princeton University Press, Princeton, N.J. 1970.

[25] R.S. Strichartz, $L^{p}$ estimates for integral transforms, Trans. Amer. Math. Soc. 136 (1969), 33-50.

[26] J. Toft, K. Johansson, S. Pilipovié, N. Teofanov, Sharp convolution and multiplication estimates in weighted spaces, Anal. Appl. 13(5) (2015), 457-480.

[27] H. Triebel, Theory of Function Spaces, Monographs in Math. Vol. 78, Birkhäuser-Verlag, Basel-Boston-Stuttgart, 1983.

[28] B. Wang, H. Hudzik, The global Cauchy problem for the NLS and NLKG with small rough data, J. Differential Equations, 232 (2007), $36-73$.

[29] B. Wang, Z. Huo, C. Hao, Z. Guo, Harmonic Analysis Method for Nonlinear Evolution Equations I, Hackensack, NJ: World Scientfic, 2011. 